\title{
A posteriori assessment of algebraic scalar dissipation models for RANS simulation of premixed turbulent combustion
}

DOI:

10.1007/s10494-017-9824-z

\section{Document Version}

Accepted author manuscript

Link to publication record in Manchester Research Explorer

Citation for published version (APA):

Ahmed, U., \& Prosser, R. (2017). A posteriori assessment of algebraic scalar dissipation models for RANS simulation of premixed turbulent combustion. Flow, Turbulence and Combustion. https://doi.org/10.1007/s10494017-9824-z

\section{Published in:}

Flow, Turbulence and Combustion

\section{Citing this paper}

Please note that where the full-text provided on Manchester Research Explorer is the Author Accepted Manuscript or Proof version this may differ from the final Published version. If citing, it is advised that you check and use the publisher's definitive version.

\section{General rights}

Copyright and moral rights for the publications made accessible in the Research Explorer are retained by the authors and/or other copyright owners and it is a condition of accessing publications that users recognise and abide by the legal requirements associated with these rights.

\section{Takedown policy}

If you believe that this document breaches copyright please refer to the University of Manchester's Takedown Procedures [http://man.ac.uk/04Y6Bo] or contact uml.scholarlycommunications@manchester.ac.uk providing relevant details, so we can investigate your claim.

\section{OPEN ACCESS}




\title{
A posteriori assessment of algebraic scalar dissipation models for RANS simulation of premixed turbulent combustion
}

\author{
Umair Ahmed · Robert Prosser
}

Received: date / Accepted: date

\begin{abstract}
This paper examines the effects of scalar dissipation rate modelling on mean reaction rate predictions in turbulent premixed flames. The sensitivity of the mean reaction rate is explored by using different closures for scalar dissipation and the sensitivity of the scalar dissipation models themselves is also examined with respect to their defining constants. The influence of different scalar dissipation models on the flame location and mean velocities is reported and compared with experimental results. The predicted reaction rate is found to be sensitive to the choice of closure used for scalar dissipation and also to the respective constants used in the scalar dissipation models. It is also found that the scalar dissipation

Umair Ahmed

School of Mechanical, Aerospace and Civil Engineering, University of Manchester, M13 9PL, $\mathrm{UK}$

E-mail: umair.ahmed@manchester.ac.uk

Robert Prosser

School of Mechanical, Aerospace and Civil Engineering, University of Manchester, M13 9PL, UK

E-mail: robert.prosser@manchester.ac.uk
\end{abstract}


models involving chemical and turbulent time scales yield a more physically plausible reaction rate when compared with the scalar dissipation models relying only on the turbulent time scale.

Keywords Algebraic scalar dissipation models · Flame turbulence interaction · RANS simulation of premixed turbulent combustion - Premixed turbulent combustion · Turbulence scalar interaction

\section{Introduction}

Accurate prediction of the mean reaction rate using computational methods remains a challenge for premixed turbulent combustion, and usually requires the use of statistical methods. There is a strong coupling between turbulence and chemistry in premixed flames and several modelling strategies have been developed to account for their interaction. In many cases, the total aerothermochemistry of the flow can be described via a Probability Density Function (PDF) and a multidimensional PDF can in principal be obtained by a PDF transport equation [31,59]. This procedure circumvents a number of modelling assumptions and consequently produces quite general reaction rate models. However the method requires a closure for the molecular diffusion terms which remains a modelling challenge [31]. Conditional Moment Closure (CMC) is another method which provides an alternative strategy for closing the reaction rate [39]. This method was originally developed for non-premixed combustion $[16,51]$ and recently has been extended to account for premixed combustion $[5,4,49]$. One of the major challenges encountered by the CMC approach in premixed combustion is the modelling of the conditional scalar dissipation rate [43]. 
In the case of a high Damköhler number limit, the closure of the reaction rate is simplified under the thin flamelet assumption. In the thin flamelet approach the flame is modelled as a sheet (an ensemble of laminar flames) separating products from reactants $[13,15,66]$, in which all of the effects of the combustion chemistry and related transport effects are restricted. Thus it becomes possible to approximate the themochemistry via a single Presumed Probability Density Function (PPDF) of a reaction progress variable, consisting of a double delta function representing the unburnt and burnt mixtures respectively [14]. This approximation leads to simple closures for a number of turbulence related terms. Several modelling approaches have been developed by using the flamelet assumption and PPDF framework and include; laminar flamelets [10,28,41]; the $G$-equation [32]; scalar dissipation rate and flame turbulence interaction based approaches $[2,3,12,40]$ and flame surface density models $[18,60]$.

One of the most widely used approaches for thin flamelets is the scalar dissipation $\left(\widetilde{\epsilon}_{c}\right)$ approach. Scalar dissipation in premixed combustion represents the average rate at which hot products and cold reactants mix on the flame surface to sustain combustion [20]. Several scalar dissipation rate models of varying complexity have been proposed, and range from a complete transport equation to simple algebraic models. The algebraic scalar dissipation rate based modelling approaches have been used in previous studies by Dong et al [25], Ahmed and Prosser [2] and Kolla and Swaminathan [41] via the Reynolds Averaged Navier Stokes (RANS) framework and by Langella et al [45] via Large Eddy Simulation (LES). In a recent study by Ahmed and Prosser [2] the leading order scalar dissipation transport equation has been used to close the reaction rate and the performance of the algebraic scalar dissipation model proposed by Kolla et al [40] is also compared 
with the predictions from the leading order scalar dissipation transport equation. Furthermore Ahmed and Prosser [2] have also compared the results of the scalar dissipation rate based modelling approach with the flame surface density based modelling approach and concluded that the scalar dissipation rate based modelling approach leads to more physically plausible results. It has also been discussed in [2] that the algebraic scalar dissipation models can lead to slight over prediction of reaction rate as the flow history effects of the strain field are not included in the algebraic closures. The aim of this study is to assess the performance of different algebraic scalar dissipation models for RANS in the corrugated and thin reaction zone combustion regimes using the experimental data of Pitz and Daily $[55,56]$ and Chen et al [22]. The models studied are proposed by Swaminathan and Bray [67], Kolla et al [40], Borghi and co-workers [48,50] and Kuan et al [44].

The paper is organised as follows: in the next section we briefly describe the models used. The test cases and the numerical procedure used for the simulations are presented in section 3 , followed by the discussion of results. The conclusions are summarised in the last section.

\section{Mathematical background}

In case of the simplified chemical mechanism $R \rightarrow P$, a transport equation for the Favre averaged progress variable $\widetilde{c}$ (a normalised product mass fraction) is solved along with Favre averaged mass and momentum equations. The transport equation for the Favre averaged progress variable can be written as :

$$
\frac{\partial \bar{\rho} \widetilde{c}}{\partial t}+\frac{\partial \bar{\rho} \tilde{u}_{i} \widetilde{c}}{\partial x_{i}}=-\frac{\partial \overline{\rho u_{i}^{\prime \prime} c^{\prime \prime}}}{\partial x_{i}}+\overline{\dot{\omega}}_{c}
$$


where closures for the mean reaction rate $\overline{\dot{\omega}}_{c}$ and the turbulent transport $\overline{\rho u_{i}^{\prime \prime} c^{\prime \prime}}$ are needed (double primes denote the Favre fluctuations). Under the Bray Moss Libby (BML) limit [15], the variance of the progress variable is closed as :

$$
\widetilde{c^{\prime \prime 2}}=\widetilde{c}(1-\widetilde{c}) .
$$

Relaxing the BML limit, some algebraic scalar dissipation models instead employ a transport equation for $c^{\prime \prime 2}$ :

$$
\frac{\partial \bar{\rho} c^{\prime \prime 2}}{\partial t}+\frac{\partial \bar{\rho} \widetilde{u}_{i} c^{\prime \prime 2}}{\partial x_{i}}=-\frac{\partial \overline{\rho u_{i}^{\prime \prime} c^{\prime \prime 2}}}{\partial x_{i}}-2 \bar{\rho} \widetilde{\epsilon}_{c}-2 \overline{\rho u_{i}^{\prime \prime} c^{\prime \prime}} \frac{\partial \widetilde{c}}{\partial x_{i}}+2 \overline{c^{\prime \prime} \dot{\omega}_{c}^{\prime \prime}} .
$$

The turbulent transport in Eq. 1 and Eq. 3 is typically modelled by a gradient diffusion model, despite the fact that countergradient diffusion may occur in premixed flames of practical interest [46]. The reasoning behind the model choice is twofold: (1) the uncertainty associated with closures embodying countergradient effects introduce additional uncertainty into the calculations, and typically have only small additional benefit for lower levels of turbulence intensities (i.e. wrinkled and corrugated flamelet regimes) $[17,70]$, and; (2) the influence of scalar dissipation rate modelling on the reaction rate closure (which is the main focus of the current work) can be studied by using the gradient diffusion approach [40,62]. The source terms in Eq. 1 and Eq. 3 are closed under the high Reynolds and Damköhler number limit as [12]:

$$
\overline{\dot{\omega}_{c}} \simeq \frac{2}{2 C_{m}-1} \bar{\rho} \widetilde{\epsilon}_{c}
$$

and :

$$
\overline{\dot{\omega}_{c}^{\prime \prime} c^{\prime \prime}}=\left(C_{m}-\widetilde{c}\right) \overline{\dot{\omega}_{c}}
$$

where $\widetilde{\epsilon}_{c}$ is the scalar dissipation $\left(\bar{\rho} \widetilde{\epsilon}_{c}=\overline{\rho \alpha \nabla c^{\prime \prime} . \nabla c^{\prime \prime}}\right), \alpha$ represents the diffusivity of the progress variable and $C_{m}$ is a model constant with a value of 0.7 in hydro- 
carbon flames [11]. The problem of modelling the reaction rate is thus reduced to that of modelling the scalar dissipation rate.

There are several approaches available in the literature to close $\widetilde{\epsilon}_{c}$; one approach is to model $\widetilde{\epsilon}_{c}$ algebraically, another is to use a modelled $\widetilde{\epsilon}_{c}$ transport equation. Here we adopt the algebraic scalar dissipation modelling approach, a number of the more widely used models are discussed in the following subsection.

2.1 Algebraic scalar dissipation models

Many algebraic models have been developed based on the leading order components of the scalar dissipation transport equation [9,48,67]. A generic form for these models is :

$$
\widetilde{\epsilon}_{c} \simeq\left(T_{1}+T_{2}\right) T_{3},
$$

where $T_{1}$ represents the effects of dilatation, $T_{2}$ represents the effects of flame turbulence interaction and $T_{3}$ represents the combined effects of reaction rate, dissipation and diffusion processes. In this work we use the algebraic scalar dissipation models proposed by Kolla et al [40] (SDR-1), Swaminathan and Bray [67] (SDR-2), Borghi and co-workers [8,48,50] (SDR-3) and Kuan et al [44] (SDR-4). The exact expressions for the terms in Eq. 5 for the different models are given in table 1.

\subsubsection{SDR-1 model}

The SDR-1 model includes the effects of heat release and Damköhler number on flame turbulence interaction (represented by $\overline{\rho \alpha \nabla c^{\prime \prime} \cdot \mathbf{S}^{\prime \prime} \cdot \nabla c^{\prime \prime}}[3]$, where $\mathbf{S}$ is the 
strain rate). The local Damköhler number $\left(D a_{L}\right)$ in table 1 is defined as :

$$
D a_{L}=\frac{u_{L}^{0} \widetilde{k}}{\delta_{L}^{0} \widetilde{\epsilon}}
$$

and $K_{c}^{*} \approx 0.85 \tau\left(\tau=\left(T_{P}-T_{R}\right) / T_{R}\right.$ is the heat release parameter $)$ for hydrocarbon flames. $u_{L}^{0}$ and $\delta_{L}^{0}$ in Eq. 6 represent the unstrained laminar flame speed and the thermal flame thickness, while $\widetilde{k}$ and $\widetilde{\epsilon}$ represent the turbulent kinetic energy and turbulent dissipation rate respectively. The empirical scaling factors $C_{3}$ and $C_{4}$ control the effects of turbulent straining and chemical straining, and are given by [41]:

$$
C_{3}=1.5\left(\frac{\sqrt{K a_{L}}}{\left(1+\sqrt{K a_{L}}\right)}\right) \quad \text { and } \quad C_{4}=\frac{1.1}{\left(1+K a_{L}\right)^{0.4}},
$$

where $K a_{L}$ is the local Karlovitz number defined as :

$$
K a_{L}=\left(u^{\prime} / u_{L}^{0}\right)^{3 / 2}\left(\delta_{L} / l_{t}\right)^{1 / 2}
$$

In Eq. $8 \delta_{L}$ is the Zeldovich thickness $\delta_{L}=\delta_{L}^{0} /\left(2(1+\tau)^{0.7}\right), u^{\prime}$ and $l_{t}$ in Eq. 8 are defined as :

$$
u^{\prime}=\sqrt{\frac{2 \widetilde{k}}{3}} \text { and } l_{t}=\frac{u^{\prime 3}}{\widetilde{\epsilon}} .
$$

The model constant $\beta^{\prime}$ in the SDR-1 model takes the value of 6.7 under the thin flame front assumption (large Damköhler number). However, there is some uncertainty in this value, with higher levels of turbulence requiring an increase of $\beta^{\prime}$ to reduce over estimates of $\widetilde{\epsilon}_{c}[52,53]$. In the past variants, SDR-1 has been used in conjunction with Eq. $3[41,42]$ and with the strict BML limit [25]. The effects of this variation are also explored in detail in the following sections.

\subsubsection{SDR-2 model}

In the SDR-2 model the constants $C_{D_{c}}$ and $C_{\epsilon_{c}}$ are taken to be 0.24 and 0.1 respectively and $\widetilde{c^{\prime \prime 2}}$ is closed via the standard BML approximation. The constant 
$C_{D}$ in SDR-2 model controls the effects of flame turbulence interaction which itself is a strong contributor to scalar dissipation [3]; Swaminathan and Bray [67] argue that $C_{D}=0.25$ based on the DNS data of a planar flame. In the results section later in the paper, the effects of varying $C_{D}$ on the RANS solution is explored.

\subsubsection{SDR-3 model}

The SDR-3 model is similar to the SDR-2 model; the only difference between the two models is the absence of the dilatation effects in the SDR-3 model. Dong et al [25] argue that $C_{D}$ implicitly includes the effects of dilatation and that the scalar dissipation should not show any dependence on Damköhler or Reynolds number. The sensitivity of the model to variations in the value of $C_{D}$ is explored later in the paper.

\subsubsection{SDR-4 model}

The SDR-4 model includes the effects of small scale turbulence, heat release and reaction rate via a fractal based approach. The constant $C_{\phi}^{*}$ in SDR-4 model takes the value $C_{\phi}^{*}=1.2[47]$. The value of $C_{\phi}$ controls the flame turbulence interaction and usually takes the value $C_{\phi}=4[44] . R e_{t}$ in the SDR-4 model represents the turbulent Reynolds number and is defined as :

$$
R e_{t}=\frac{u^{\prime} l_{t}}{\nu_{u}}
$$

where $\nu_{u}$ is the viscosity of the unburnt mixture. The sensitivity of the SDR-4 model to the changes in $C_{\phi}$ are explored in the following sections. 


\section{Test configurations and numerical procedure}

In order to assess the performance of the algebraic scalar dissipation models, RANS simulations of two different flames have been carried out; a premixed flame in a dump combustor configuration and a premixed Bunsen flame configuration. These flames lie in different parts of the regime diagram shown in figure 1.

The calculations for this work have been performed using the unstructured finite volume code Code_Saturne [6] (see http://www.code-saturne.org). The code solves the Navier-Stokes equations for Newtonian low Mach number variable density flows with a fractional step method based on a prediction-correction algorithm for pressure/velocity coupling (SIMPLEC) and a Rhie and Chow interpolation to avoid pressure oscillations. The code uses a second order central differencing scheme for spatial gradients; the time integration is undertaken via an Euler explicit scheme. This code has previously been validated and used for several industrial and academic studies ranging from simulations of incompressible flows $[1,7$, $33,34,35]$ to low Mach number variable density reacting flows [2, 25, 30, 37].

\subsection{Dump combustor}

The experiments performed by Pitz and Daily $[55,56]$ are used as the first test case. This case is representative of typical geometries found in gas turbine engines. The case under consideration is a propane-air premixed flame with an equivalence ratio $(\phi)$ of 0.57 . The experiment consists of a rectangular premixing region followed by a smooth contraction. There follows a step expansion into the combustion chamber and a quenching water spray filled converging exit region. Note that in the simulations, the smooth contraction at the inlet is omitted and no attempt is 
made to represent the effects of the spray at the exit. The flame is stabilised in a turbulent-free shear layer formed at the backward facing step shown in figure 2. The converging exit region is simulated in order to move the outlet boundary condition sufficiently far from the recirculation region adjacent to the step. The mean inlet values for the bulk velocity, pressure, temperature and thermochemical properties are given in table 2. The Reynolds number based on bulk velocity and step height is 22100. Further details of the experiment and measuring techniques used can be found in $[55,56]$. This test case has been previously studied by Weller et al [71] and Fureby [27] via LES, Ahmed and Prosser [2] by using RANS and by Tangermann et al [68] using both LES and RANS.

A no-slip condition is applied to the velocity at the walls, while all the scalars are treated with zero Neumann conditions. Symmetry conditions are used in the transverse direction. Similar boundary conditions have been used in the earlier studies $[2,71]$ for the same rearward facing step. The simulations are run until a statistically steady flow is achieved.

\subsection{Bunsen flame}

The second test case considered is one of the piloted stoichiometric methane-air Bunsen flames studied by Chen et al [22]. This flame has been extensively investigated in several previous studies via RANS $[5,32,42,61,62]$ and via LES $[23,24$, $45,54]$ using different reaction rate closure strategies. In this flame, stoichiometric methane air mixture is injected through a fuel nozzle of diameter $D=12 \mathrm{~mm}$, surrounded by a laminar pilot flame of a stoichiometric methane-air mixture with a pilot diameter of $D_{p}=68 \mathrm{~mm}$. The bulk mean velocity $\left(u_{0}\right)$ in the fuel nozzle is 30 
$\mathrm{m} / \mathrm{s}$ and the jet exit Reynolds number based on $u_{0}$ is 24000 . The centreline value of turbulent kinetic energy at the burner exit, $k_{0}$, is $3.82 \mathrm{~m}^{2} / \mathrm{s}^{2}$. The experimental setup is shown in figure $3(\mathrm{a})$. In the experiment, the pilot stream was cooled by a water circuit. This presents a difficulty in modelling this flame with an adiabatic flamelet model. In order to overcome this problem, the strategy proposed by Kolla and Swaminathan [42] is used. According to this formulation the sub adiabatic temperatures at the pilot boundary are handled by the Favre averaged transport equation for total enthalpy $(\widetilde{h})$ :

$$
\frac{\partial \bar{\rho} \widetilde{h}}{\partial t}+\frac{\partial \bar{\rho} \tilde{u}_{i} \widetilde{h}}{\partial x_{i}}=-\frac{\partial \overline{\rho u_{i}^{\prime \prime} h}}{\partial x_{i}}
$$

where the turbulent transport term is closed by a gradient diffusion model. The total enthalpy is defined as the sum of chemical and sensible enthalpies of the mixture [42]:

$$
\widetilde{h}=C_{p_{m i x}}\left(\widetilde{T}-T_{R}\right)+\Delta h_{f_{m i x}}^{0},
$$

where $C_{p_{m i x}}=\sum \tilde{Y}_{i} C_{p_{i}}$ and $\Delta h_{f_{m i x}}^{0}=\sum \tilde{Y}_{i} \Delta h_{f_{i}}^{0} \cdot C_{p_{i}}$ and $\Delta h_{f_{i}}^{0}$ are the specific heat and formation enthalpy of species $i$, respectively. The temperature is calculated as $\widetilde{T}=T_{R}+\left(\widetilde{h}-\Delta h_{f_{m i x}}^{0}\right) / C_{p_{m i x}}$ and the mixture density is calculated as $\bar{\rho}=p W_{m i x} /(\Re \widetilde{T})$, where $W_{m i x}$ and $\Re$ are the molecular weight of the mixture and the universal gas constant respectively. The formulation assumes that the flame brush does not interact with the entrainment air and hence has no influence on the chemical composition of the flame. A transport equation for Favre averaged mixture fraction $(\widetilde{\xi})$ is additionally solved to account for the effects of entrainment. Inlet values for $\widetilde{\xi}$ are taken as 0 and 1 in the ambient and reacting regions, 
respectively. The values of $C_{p_{m i x}}, \Delta h_{f_{m i x}}^{0}$ and $W_{m i x}$ are calculated as [42]:

$$
\begin{aligned}
C_{p_{\text {mix }}} & =\widetilde{\xi} C_{p_{\text {reac }}}+(1-\widetilde{\xi}) C_{p_{a i r}}, \\
\Delta h_{f_{\text {mix }}}^{0} & =\widetilde{\xi} \Delta h_{f_{\text {reac }}}^{0}+(1-\widetilde{\xi}) \Delta h_{f_{a i r}}^{0}, \\
W_{\text {mix }} & =\widetilde{\xi} W_{\text {reac }}+(1-\widetilde{\xi}) W_{a i r}
\end{aligned}
$$

where subscripts reac and air represent the fluid property in the reacting mixture and in the co-flow air, respectively. The air is assumed only to contain $\mathrm{O}_{2}$ and $N_{2}$, hence $\Delta h_{f_{a i r}}^{0}=0$. The values for $C_{p_{\text {reac }}}, \Delta h_{f_{\text {reac }}}^{0}$ and $W_{\text {reac }}$ are tabulated in a look up table as a function of the progress variable. The look up table is generated by using a planar unstrained laminar premixed flame of a stoichiometric methane-air mixture computed using Cantera [29] and the GRI 3.0 mechanism [65] with the mixture averaged diffusivity formulation. The progress variable in this case is defined as $\widetilde{c}=1-Y_{F} / Y_{F_{u}}$ ( $Y_{F}$ is the fuel mass fraction and $Y_{F_{u}}$ is the fuel mass fraction in the unburnt mixture).

A two dimensional axisymmetric domain is used to simulate the Bunsen flame. The simulated domain size along with the boundary conditions are shown in figure 3(b). The domain has been made large enough in the radial and axial directions such that the domain boundaries do not influence the flame structure. As in the dump combustor case, the simulations are run until a statistically steady flow is achieved.

\section{Results and discussion}

It has been shown in earlier work $[47,62]$ that the algebraic scalar dissipation models are most sensitive to the choice of the constants used for the term representing 
flame turbulence interaction $\left(\overline{\rho \alpha \nabla c^{\prime \prime} \cdot \mathbf{S}^{\prime \prime} \cdot \nabla c^{\prime \prime}}\right)$. Hence the models described in section 2 have been used with different constants for the flame turbulence interaction term. In most models there is no universal value for this constant $[25,47,53,57$, $62,67]$ as it is dependant on the local flow conditions including local turbulence, flame properties and local strain rates. The changes in the different values for the constants for all the models used in this study are presented in table 3 .

\subsection{Dump combustor calculations}

\subsubsection{Isothermal calculation}

Isothermal calculations for this configuration have been performed to assess the suitability of the turbulence model used. Inlet velocity and turbulence intensity profiles for the simulations are taken from the experiment of Pitz and Daily [55, 56], and are provided in appendix-A. Grid sensitivity study presented in appendixB has demonstrated that a grid with 20000 cells yields grid independent results. The standard $k-\epsilon$ model proposed by Jones and Launder [36] is used along with the standard wall function for near wall treatment, and the results for velocity are shown in figure 4 . The recirculation region is captured slightly incorrectly, causing a small discrepancy in the predicted flow velocity further downstream of the backward facing step, commensurate with the shorter separation typically predicted by two equation models [69]. The root mean squared velocity $\left(u_{r m s}\right)$ predictions from the $k-\epsilon$ model are shown in figure 5. In this case the regions of high turbulence occur in the shear layer and broaden with downstream distance from the backward facing step. It can be seen that the $u_{r m s}$ predictions match the experimental and LES results reasonably well near the backward facing step, and are under predicted 
in the upper half of the dump combustor $(y / h>0)$ for the regions away from the backward facing step. Again this is consistent with the typical predictions of the two equation models [69]. The size of the recirculation bubble predicted by the $k-\epsilon$ model is shown in figure 6 . The white line in figure 6 denotes the zero velocity region, which indicates the length of the recirculation bubble. It can be seen that the size of the recirculation bubble is $x_{r} / h \approx 6.7$, which is slightly smaller than the experimental value of $x_{r} / h \approx 7.0$. These results are consistent with those of Furbo [26] and Ahmed and Prosser [2] for this geometry and of Klein et al [38] for a generic backward facing step. It has been shown in earlier studies that $k-\epsilon$ model performs well for similar configurations when compared with other models $[2,38]$. In the specific case of this dump combustor Furbo [26] has shown that the $k-\epsilon$ model performs well when compared with second moment closures and other linear eddy viscosity models. Hence the standard $k-\epsilon$ model is used for the reacting flow simulations.

\subsubsection{Reacting flow calculations}

In the reacting case, the incoming fluid contains cold premixed reactants which mix with the hot products in the shear layer formed behind the backward facing step and subsequently burn. The shear layer anchors the flame to the backward facing step.

A special treatment for all of those algebraic scalar dissipation models considered here is needed to suppress the near wall reaction rate, as these models predict reaction in the boundary layer formed at the top wall of the dump combustor. To rectify the problem we use the quenching model proposed by Catlin and Lindstedt [19], which has also been used for this configuration in previous studies $[2,68]$. The 
model suppresses the reaction rate if the progress variable falls below a quenching value $\widetilde{c}_{q}$, which in case of adiabatic conditions corresponds to a quenching temperature $T_{q}=\widetilde{c}_{q}\left(T_{P}-T_{R}\right)+T_{R}$. In the calculations here the reaction is suppressed if the temperature falls below $T_{q}=300 K[2,68]$.

First we compare the predicted length of the recirculation zone in the wake of the backward facing step. The length of the recirculation zone in the reacting case is shorter than in the cold flow case and determines the anchor location for the flame. Table 4 shows the predicted lengths for the recirculation zone from different models. The length of the recirculation zone is measured in the same way as it is done for the isothermal simulation presented in section 4.1.1. Note that the recirculation length is sensitive to the choice of model used and also to the changes in the values of the constants used in $\widetilde{\epsilon}_{c}$ models. It is also seen that the choice of closure used for $\widetilde{c^{\prime \prime 2}}$ does not alter the length of the recirculation zone for all the values of $\beta^{\prime}$ used in the SDR-1 model. The main reason for the change in the recirculation length is due to the variation in the prediction of the reaction rate by different models, which is discussed later on in this section.

Figure 7 shows the mean velocity predicted when using different scalar dissipation models. Generally the predicted velocity shows similar trends for all the models used. SDR-1.2 and SDR-1.6 predict identical velocity profiles for the dump combustor, hence the results from the SDR-1.2 model only are presented. At $x / h=1$ all the models tend to over predict the velocity. This is due the high reaction rate prediction in this zone as shown in figures 9 and 11. Figure 11 shows that the predicted reaction zones are attached to the backward facing step, which is contrary to the earlier findings $[2,27]$, where it was shown that the reaction zone is anchored in the recirculation zone further downstream. The high reaction rate 
prediction in this region is due to the dependence of the models on the turbulent time scale. $\overline{\dot{\omega}_{c}} \rightarrow \infty$ as $\widetilde{k} \rightarrow 0$ at the wall and the attachment point moves to the edge of the step as a result. The sensitivity of the predicted velocity to the choice of the constants used in the models can be seen in figure 7 at the locations downstream of the backward facing step. The velocity prediction is most sensitive at $x / h=7$ as shown in figure $7 \mathrm{~d}$. One of the reasons for this difference in the velocity prediction from different models is due to the treatment of the flame turbulence interaction term, as SDR-1 model accounts for the change in the source and sink nature of flame turbulence interaction while the other models only predict it as a source term. Another reason for this difference is the inclusion of dilatation effects due to heat release in SDR-1 and SDR-2 models as shown in table 1. These effects have a significant influence on the reaction rate prediction as discussed later on in this section.

Table 5 shows the influence of change in $\beta^{\prime}$ on the scaling factors $C_{3}$ and $C_{4}$ in the SDR- 1 model. In this case $\beta^{\prime}$ is changed by approximately $17.7 \%$ and leads to a significant change in the values for $C_{3}$ and $C_{4}$. The percentage difference in the scaling factors increases as the distance from the backward facing step increases, which suggests that $C_{3}$ and $C_{4}$ change in a non linear manner when the value of $\beta^{\prime}$ is altered. This implies that the value of $\beta^{\prime}$ scales as the local values of $R e_{t}$ and $K a$ of the flow. The sensitivity of the SDR-1 model to $\beta^{\prime}$ is investigated further in the Bunsen flame calculations.

Figure 8 shows the root mean squared velocity predicted by the SDR-1.2, SDR-2.1, SDR-3.3 and SDR-4.1 models. Generally root mean squared velocity predictions are similar for all the scalar dissipation models considered in this study. Similar to the non reacting case the regions of high turbulence intensity occur in 
the shear layer and widen with the distance away from the backward facing step. $u_{r m s}$ predicted by all the scalar dissipation models is in reasonable agreement with the experimental and LES data at $x / h=1$. Further downstream of the backward facing step at $x / h=3$ the $u_{r m s}$ values are slightly under predicted by all models for the lower part of the dump combustor $(y / h<0)$. At $x / h=5$ and $x / h=7$ the the predictions for $u_{r m s}$ improve for all scalar dissipation models.

Variation in the prediction of reaction rates from the SDR-1.2, SDR-2.1, SDR3.3 and SDR-4.1 models at several locations downstream of the rearward facing step can be seen in figure 9 . These variations can be explained by differences in the underlying assumptions for different models. Models relying on chemical and turbulent time scales (SDR-1and SDR-2) predict a lower reaction rate near the backward facing step while models relying on just the turbulent time scale tend to predict a significantly higher reaction rate. The SDR-2 model predicts a higher reaction rate at $x / h=5$ and $x / h=7$ when compared with the SDR-1 model as the flame turbulence interaction closure used in this model does not account for the change in the alignment of the strain rate eigenvectors with local variations in heat release and turbulence. The predictions of flame turbulence interaction from different models is shown in figure 10. Note that all the scalar dissipation models except the SDR-1 model predict a positive value for flame turbulence interaction, whereas the SDR-1 model predicts a variation of flame turbulence interaction and changes from a source to a sink term as the distance from the step increases. The sink nature of the predicted flame turbulence interaction opposes the effects of dilatation in the SDR-1 model and decreases the overall reaction rate. Whereas the source nature of the predicted flame turbulence interaction in SDR-2, SDR-3 and SDR-4 models leads to an overall increase in the predicted reaction rate. The 
reaction rates predicted by the SDR-1 model at $x / h=5$ and $x / h=7$ are in reasonable agreement with the earlier results of Ahmed and Prosser [2] obtained via a full flame turbulence interaction transport equation.

Figure 11e shows a Schlieren photograph of the reacting flow from the experiment. The brightest areas in this figure show the highest reaction rate regions. A similar behaviour for the predicted reaction rate from SDR-1.2 and SDR-2.1 models can be seen in figure 11a and figure 11b. This behaviour of SDR-1 and SDR-2 models is expected as these models rely on similar modelling assumptions and include a turbulent and chemical time scale, whereas the SDR-3 and SDR-4 models only rely on a turbulent time scale and predict a similar reaction rate as shown in figures 11c-11d. A high reaction rate is predicted by the SDR-2.1 model through out the domain when compared with the SDR-1.2 model. This is due to the difference in the closure for flame turbulence interaction used in these models. The flame turbulence interaction mainly acts as a sink term in the flame predicted by the SDR-1.2 model (figure 10) which counteracts the effects of dilatation, while the SDR-2.1 model always predicts a positive flame turbulence interaction (figure 10) thus leading to an enhancement of the dilatation effects leading to a higher reaction rate prediction. Note that the models relying on the turbulent time scale only predict a high reaction zone near the backward facing step and then the reaction rate tends to decrease (figures 11c- 11d), whereas the models relying on turbulent and chemical time scales tend to predict a more uniform reaction zone (figures 11a- 11b). Figure 11a shows that the SDR-1.2 model tends to predict a reaction zone which is closest to the flame shape in the Schlieren image (figure 11e) reported in the experiment. 
Predicted flame locations can be further verified by comparing the predicted Reynolds averaged progress variable with the LES and experimental data. The Reynolds averaged progress variable in the simulations is calculated as [42]:

$$
\bar{c}=\widetilde{c}+\frac{\tau c^{\prime \prime 2}}{1+\tau \widetilde{c}},
$$

where $\widetilde{c^{\prime \prime 2}}$ is approximated via the BML assumption for the SDR-2.1, SDR-3.3 and SDR-4.1 models. Figure 12 shows the comparison of the progress variable for all the algebraic scalar dissipation models used in this study. All the models tend to predict similar values for the progress variable at $x / h=0.4$ and $x / h=1.2$. Further downstream the differences in the predictions of the progress variable from different models become more apparent. The prediction of progress variable from SDR-1.2 at $x / h=3.5$ and $x / h=5.4$ slightly improves at the trailing edge of the flame while the SDR-2.1, SDR-3.3 and SDR-4.1 slightly over predict the location for the progress variable.

4.2 Bunsen flame calculations

\subsubsection{Isothermal calculation}

Isothermal flow calculations have been performed to assess the performance of the turbulence model. Simulations have been performed by using a modified form of the standard $k-\epsilon$ model, where the value of the constant $C_{\epsilon 1}$ has been changed from 1.44 to 1.6 to account for the round-jet anomaly [58]. The inlet conditions for the mean velocity and turbulent kinetic energy are taken from the experimental data of Chen et al [22], and are shown in appendix-A. The integral length scale at the inlet for the main jet is specified as $l_{t} \approx 0.0024 m$, while very small values 
$\left(1 \times 10^{-5}\right)$ for turbulent kinetic energy and turbulent dissipation are specified at the inlet for the pilot and air co-flow streams. In the case of isothermal calculations a small velocity of $0.2 \mathrm{~m} / \mathrm{s}$ has been specified at the inlet for the pilot and co-flow air streams. Grid sensitivity study presented in appendix-B has shown that grid independent results were observed for a structured non uniform axisymmetric grid of 47000 cells.

Figures 13 and 14 show the predicted mean velocity and turbulent kinetic energy profiles respectively at different locations downstream of the jet exit. It can be seen from the experimental data in figure 13 that the spreading of the jet corresponds to the development of a lateral shear layer at $y / D \approx 2.5$ (between $0.25<r / D<0.75)$. At $y / D \approx 4.5$ the maximum mean velocity decreases as the jet starts to expand in the radial direction. The profiles for turbulent kinetic energy shown in figure 14 also display the lateral shear layers which surround the central jet. The turbulent kinetic energy increases along the centre line of the jet as the distance from the inlet increases. This indicates the merging of the shear layers and thus the ending of the potential core. The simulation is able to capture these effects appropriately at all the sampling locations as shown in figures 13 and 14. Hence the $k-\epsilon$ model with $C_{\epsilon 1}=1.6$ is used for the reacting flow calculations.

\subsubsection{Reacting flow calculations}

In the reacting case the shear layer formed at the interface of the cold reactants and the pilot stream effectively anchors the flame. The unstrained laminar flame speed $\left(u_{L}^{0}\right)$ for methane at stoichiometric conditions at $298 K$ is $0.4 m / s[22]$, the thermal flame thickness $\left(\delta_{L}^{0}\right)$ is $1.436 \mathrm{~mm}$ and the kinematic viscosity $\left(\nu_{u}\right)$ is $7.0 \times$ $10^{-5} \mathrm{~m}^{2} / \mathrm{s}$. Several workers have used different values of inlet temperature for 
the pilot stream as the measurements for the radial variation of the pilot stream temperature have not been reported in the experiment; following earlier studies [42, $45]$ a value of $1950 \mathrm{~K}$ has been used here. In the case of reacting flow calculations a velocity of $1.5 \mathrm{~m} / \mathrm{s}$ has been specified for the pilot stream and a velocity of $0.2 \mathrm{~m} / \mathrm{s}$ for the co-flow air.

The SDR-1 model has been tested with $\widetilde{c^{\prime \prime 2}}$ transport equation and also with the BML closure for $c^{\pi / 2}$. It is found that slightly improved predictions are made when the $\widetilde{c^{\prime \prime 2}}$ transport equation is used in this case, hence only the results from the SDR-1 model with $\widetilde{c^{\prime \prime 2}}$ transport equation are presented in this section. The reason for this behaviour is due to the fact that the Bunsen flame lies in the thin reaction zone regime; in this regime turbulence can enter the flame structure and reduces $\widetilde{c^{\prime \prime 2}}[21]$. The contributions from the burning mode PDF become nonnegligible in the thin reaction zone regime [21] and a transport equation for $\widetilde{c^{\prime \prime}}$ allows for the relaxation of the BML limit. In the case of SDR-1 model $\beta^{\prime}=6.7$ leads to an unphysically small flame which is consistent with the earlier findings of Salehi and Bush [62]. In the Bunsen flame case the value of $\beta^{\prime}$ needs to be changed significantly to obtain a physically plausible flame height. The influence of change in $\beta^{\prime}$ on the scaling factors $C_{3}$ and $C_{4}$ can be seen in table 6 . A significant change can be observed in the values for $C_{3}$ and $C_{4}$ at all the sampling locations when the value of $\beta^{\prime}$ is altered. This observation is consistent with the dump combustor case, and implies that $\beta^{\prime}$ should be increased with an increase in the local values of $R e_{t}$ and $K a$ to obtain a physically plausible solution.

Figure 15 shows the reacting flow velocity predicted by using different scalar dissipation models. The sensitivity of scalar dissipation models to the choice of the constants used in the flame turbulence interaction term can be noted in figure 15. 
As in the dump combustor case the velocity predictions from the different variants of the SDR-2 model are higher than the experimental values. This is due to the fact that a shorter flame is predicted by the variants of the SDR-2 model. Note that the SDR-3.2 and the SDR-4.2 models predict an identical velocity filed despite the fact that these models have been developed using different physical and theoretical arguments. Velocity predictions from the SDR-1.4, SDR-3.2 and SDR-4.2 models are in reasonable agreement with the experimental data up to $y / D \approx 6.5$. Further downstream these models tend to under predict the velocity as shown in figure $15 \mathrm{~d}$.

Figure 16 shows the turbulent kinetic energy predicted by SDR-1.4, SDR-2.1, SDR-3.2 and SDR-4.2 models. In the reacting case the turbulent kinetic energy is smaller when compared with the isothermal case and increases with the distance away from the jet inlet. Also note that unlike the isothermal case the location of maximum turbulent kinetic energy moves outwards with increasing distance away from the jet. All the scalar dissipation models show similar trends for the turbulent kinetic energy at all the sampling locations. Note that while the mean velocities for these models are in reasonable agreement with the experiment, the values of the turbulent kinetic energy are over predicted at the regions near the jet inlet at $y / D \approx 2.5$ and $y / D \approx 4.5$. This over prediction has been reported in earlier RANS simulations by Kolla and Swaminathan [42] and Lindstedt and Vaos [47]. The predicted turbulent kinetic energy starts to improve further downstream of the jet inlet (at $y / D \approx 6.5$ and $y / D \approx 8.5$ ) and a reasonable agreement can be seen for all the scalar dissipation models at $y / D \approx 8.5$.

Figure 17 shows the predicted temperature profiles by using different scalar dissipation models. It can be seen that the predicted temperature profiles are sen- 
sitive to the choice of the scalar dissipation model used. The predicted temperature profiles from all the scalar dissipation models are in good agreement in middle of the jet and the shear layer at $y / D \approx 2.5$, but an over prediction of temperature can be seen in the pilot region $(r / D>0.75)$. This discrepancy in temperature is due to the lack of proper information for boundary conditions for temperature and has been reported in earlier studies $[32,42,62,63]$. The discrepancy between the predicted and experimental temperature field decreases as the distance from the jet exit increases as shown in figure 17. Note that SDR-3.2 and SDR-4.2 models predict identical temperature profiles throughout the flame, which is consistent with the velocity predictions from these models. At $y / D \approx 6.5$ and $y / D \approx 8.5$ high temperature predictions from the SDR-2.1 model can be noticed in the core of the jet as a shorter flame is being predicted by the SDR-2.1 model and results in an early increase in temperature in the core of the jet.

The predicted reaction rate from different scalar dissipation models are presented in figure 18 and figure 20. It can be seen in figures 18 and 20 that the SDR-3.2 and SRD-4.2 models predict almost similar reaction rates for the Bunsen flame case. Generally the reaction rate predicted by all the models decreases as the distance from the jet increases. A high reaction rate is noticed for SDR-2.1, SDR-3.2 and SDR-4.2 models near the jet exit at $y / D \approx 2.5$, whereas the SDR-1.4 model predicts a much smaller reaction rate in this region as shown in figure 18a. High reaction rate prediction by SDR-2.1, SDR-3.2 and SDR-4.2 models is due to an infinite growth of flame turbulence interaction term as shown in figure 19 which always acts as a source term for scalar dissipation in these models - this is not always the case as the flame induced turbulence scalar interaction can destroy the flame due to local changes in the alignment of the strain rate with respect 
to the flame gradients. The SDR-1.4 model is able to predict the change in the source and sink nature of the flame turbulence interaction term across the flame as shown in figure 19. Note that the reaction zone predicted by all the models starts to move towards the centre of the jet as the distance from the jet exit increases. This happens as the mixing layers formed by the reactants and products at the outer regions of the jet start to merge together. The complete merger of the product and reactant streams marks the height of the flame in case of premixed Bunsen flames [64], and the location of the complete merger of these two streams is sensitive to the choice of the scalar dissipation models used as shown in figure 20. Note that the reaction rate prediction in the core of the jet by the SDR-2.1 model increases at a much higher rate when compared with the other models. This is due to a higher flame propagation speed being predicted by the SDR-2.1 model which consequently leads to a prediction of smaller flame height.

The location of reaction zones predicted by different scalar dissipation models can be seen in figure 20. It can be noticed in figure 20b that the SDR-2.1 model predicts the shortest reaction zone and consequently leading to a shorter predicted flame. SDR-3.2 and SDR-4.2 models predict the longest reaction zones which are almost identical to each other (figure 20c and figure 20d); this is in agreement with the predicted temperature and velocity profiles discussed earlier. Note that the models relying on both the chemical and turbulent time scales lead to a physically plausible reaction zone while the models relying on just the turbulent time scale lead to a high reaction zone in the shear layer near the jet exit and then a sudden decrease in the reaction rate is observed down stream of the jet. SDR-1.4 model predicts the most physically plausible reaction zone in this case (figure 20b), as it 
accounts for the local production or destruction of scalar gradients by turbulence in the scalar dissipation approximation.

The turbulent flame brush thickness predicted by different scalar dissipation models is compared with the experimental data in figure 21. The turbulent flame brush thickness in this case is calculated as :

$$
\delta_{t}=\left(\left.\frac{\partial \bar{c}}{\partial r}\right|_{\max }\right)^{-1}
$$

where the Reynolds averaged progress variable in the simulations is calculated via Eq. 14. Figure 21 shows that the flame brush thickness increases linearly with the distance away from the jet exit which implies that the flame brush thickness in this case is determined by the local turbulence [22]. All the scalar dissipation models used here tend to capture the correct trend of the turbulent flame thickness at all sampling locations except the SDR-2.1 model; which predicts a substantially higher flame brush thickness away from the jet exit at $y / D \approx 6.5$ and $y / D \approx 8.5$, while all the other models except SDR-1.4 tend to slightly under predict the flame brush thickness as shown in figure 21. The large flame brush thickness predicted by the SDR-2.1 at the down stream locations is a result of the high reaction rate prediction near the jet exit as shown in figure 20 .

\section{Summary and Conclusions}

In this study, the algebraic scalar dissipation models proposed by Kolla et al [40] (SDR-1), Swaminathan and Bray [67] (SDR-2), Borghi and co-workers [48,50] (SDR-3) and Kuan et al [44] (SDR-4) have been compared on two different test cases; a dump combustor (corrugated flamelet regime) and a Bunsen flame (thin reaction zone regime). Initial isothermal calculations performed with the standard 
$k-\epsilon$ model found it to work well for the dump combustor case, but required the correction proposed by Pope [58] to account for the round jet.

In view of the results obtained from flames in this study, constants involved in the algebraic scalar dissipation models are seen to play an important role, especially for the terms involving flame turbulence interaction. In the case of SDR2, SDR-3 and SDR-4 models, the constant acting on the turbulent time scale ratio plays an important role in determining the mixing time scale and consequently controlling the flame propagation speed; higher values for the constant leads to a higher reaction rate prediction. The predictive capability of the SDR-1 model relies on the value of $\beta^{\prime}$, which controls all the other constants and scaling factors $\left(K_{c}^{*}\right.$, $C_{3}$, and $C_{4}$ ) used in the SDR-1 model (see table 1 and Eq. 5). Generally values close to the default value of $\beta^{\prime}$ in SDR-1 model give reasonable predictions in the wrinkled/ corrugated flamelet regime, but lead to unphysical flame behaviour in the case of thin reaction zone regime. The value of $\beta^{\prime}$ has to be increased significantly from its default value to obtain a physically plausible flame in the thin reaction zone regime. Coupling the $\widetilde{c^{\prime \prime} 2}$ transport equation to the SDR-1 model does not make a significant difference in the wrinkled/ corrugated flamelet regime, but it leads to an overall improvement of results in the thin reaction zone regime, where $\widetilde{c^{\prime \prime 2}}$ transport allows for departures from the strict BML limit, and allows contributions of the burning mode PDF to be included in the modelling strategy. The flame turbulence interaction approximation used in the SDR-1 model is able to predict the change in alignment between the strain filed and the scalar gradients; SDR-2, SDR-3 and SDR-4 models are unable to predict such changes.

Algebraic scalar dissipation models relying on the turbulent and chemical time scales (SDR-1 and SDR-2) predict a more physically plausible reaction zone in the 
corrugated flamelet and thin reaction zone regimes. It is also observed that all the algebraic scalar dissipation models predict a high non-physical reaction rate near walls. Recommendations regarding the choice of the constants used for different scalar dissipation models in wrinkled/corrugated flamelet and thin reaction zone combustion regimes are listed in table 7 . All the scalar dissipation models predict slightly erroneous results in the far wake of the flame stabilisation region in the two combustion regimes investigated in this paper. This is due to the under performance of the flame turbulence interaction approximation used in these models. Further improvement of the algebraic scalar dissipation models form part of the ongoing work.

\section{Acknowledgements}

The authors would like to thank the reviewers for their helpful and constructive comments regarding draft versions of this manuscript.

\section{Compliance with Ethical Standards}

Conflict of Interest: The authors declare that they have no conflict of interest.

\section{Appendix-A Inlet velocity and turbulence intensity profiles used in the simulations}

The inlet profiles for the simulations performed in this study are taken from the original experiments. Inlet profiles for mean velocity and turbulence intensity are given in figure 22 for the dump combustor case; they are taken from the experiments of Pitz and Daily [55]. In the case of Bunsen flame simulations, the inlet 
velocity and turbulence intensity profiles have been taken from the experiment of Chen et al [22] and are presented in figure 23.

\section{Appendix-B Grids used in the computations}

A fully structured hexahedral mesh is used for the dump combustor simulation.

Three different meshes have been tested with different refinement levels; 10000 cells (coarse), 20000 cells (medium) and 40000 cells (fine). The region near the backward facing step in the case of medium mesh is shown in figure 24a. Note that that the $k-\epsilon$ model uses a standard wall function near the walls and requires $y^{+} \approx 30$ at the wall. The results from the mesh sensitivity studies are presented in figure 25. No discernible difference can be seen in the different velocity predictions for the different meshes used; the medium mesh has been chosen for the reacting flow simulations.

Figure 24b shows the mesh in the region near air-fuel mixture inlet in the Bunsen flame case. Three different axisymmetric hexahedral meshes have been tested; 24000 cells (coarse), 47000 cells (medium) and 82000 cells (fine). Figure 26 demonstrates that the velocity predictions from the different meshes are almost identical to each other; the medium mesh has been chosen for the reacting flow simulations.

An error analysis has been carried out by using the infinity norm on different velocity profiles (not shown here) and it is found that changing the mesh from fine to coarse leads to a negligible error of less than $0.5 \%$ in the velocity field for both cases. 


\section{References}

1. Ahmed, U., Apsley, D.D., Afgan, I., Stallard, T., Stansby, P.K.: Fluctuating loads on a tidal turbine due to velocity shear and turbulence: comparison of CFD with field data. Renewable Energy 112(2017), 235-246 (2017). DOI 10.1016/j.renene.2017.05.048

2. Ahmed, U., Prosser, R.: Modelling flame turbulence interaction in RANS simulation of premixed turbulent combustion. Combustion Theory and Modelling 20(1), 34-57 (2016). DOI $10.1080 / 13647830.2015 .1115130$

3. Ahmed, U., Prosser, R., Revell, A.J.: Towards the development of an evolution equation for flame turbulence interaction in premixed turbulent combustion. Flow, Turbulence and Combustion 93(4), 637-663 (2014). DOI 10.1007/s10494-014-9557-1

4. Amzin, S., Swaminathan, N.: Computations of turbulent lean premixed combustion using conditional moment closure. Combustion Theory and Modelling 17(6), 1125-1153 (2013). DOI $10.1080 / 13647830.2013 .848382$

5. Amzin, S., Swaminathan, N., Rogerson, J.W., Kent, J.H.: Conditional moment closure for turbulent premixed flames. Combustion Science and Technology 184(10-11), 1743-1767 (2012). DOI 10.1080/00102202.2012.690629

6. Archambeau, F., Mechitoua, N., Sakiz, M.: A finite volume method for the computation of turbulent incompressible flows - industrial applications. Int. J. Finite Volumes 1, 1-62 (2004)

7. Benhamadouche, S., Laurence, D.R.: LES, coarse LES, and transient RANS comparisons on the flow across a tube bundle. International Journal of Heat and Fluid Flow 24(4), 470-479 (2003). DOI 10.1016/S0142-727X(03)00060-2

8. Borghi, R.: Turbulent premixed combustion: Further discussions on the scales of fluctuations. Combustion and Flame 80(3-4), 304-312 (1990). DOI 10.1016/0010-2180(90)901062

9. Borghi, R., Dutoya, D.: On the scales of the fluctuations in turbulent combustion. Symposium (International) on Combustion 17(1), 235-244 (1979). DOI 10.1016/S00820784(79)80025-9

10. Bradley, D., Gaskell, P., Gu, X.: Application of a Reynolds stress, stretched flamelet, mathematical model to computations of turbulent burning velocities and comparison 
with experiments. Combustion and Flame 96(3), 221-248 (1994). DOI 10.1016/00102180(94)90011-6

11. Bray, K.: Turbulent flows with premixed reactants. In: P. Libby, F. Williams (eds.) Turbulent Reacting Flows, Topics in Applied Physics, vol. 44, pp. 115-183. Springer Berlin / Heidelberg (1980)

12. Bray, K.N.C.: The interaction between turbulence and combustion. Seventeenth Symposium Symposium (International) on Combustion 17(1), 223-233 (1979). DOI 10.1016/S0082-0784(79)80024-7

13. Bray, K.N.C., Libby, P.A.: Interaction effects in turbulent premixed flames. Physics of Fluids 19(11), 1687 (1976). DOI 10.1063/1.861384

14. Bray, K.N.C., Libby, P.A., Moss, J.B.: Unified modeling approach for premixed turbulent combustion-Part I: General formulation. Combustion and Flame 61(1), 87-102 (1985). DOI 10.1016/0010-2180(85)90075-6

15. Bray, K.N.C., Moss, J.B.: A unified statistical model of the premixed turbulent flame. Acta Astronautica 4(3-4), 291-319 (1977). DOI 10.1016/0094-5765(77)90053-4

16. Bushe, W.K., Steiner, H.: Conditional moment closure for large eddy simulation of nonpremixed turbulent reacting flows. Physics of Fluids 11(7), 1896-1906 (1999). DOI $10.1063 / 1.870052$

17. Cant, R.: RANS and LES modelling of premixed combustion. In: T. Mastorakos E.and Echekki (ed.) Turbulent Combustion Modeling, pp. 63-90. Springer (2011)

18. Cant, R.S., Pope, S.B., Bray, K.N.C.: Modelling of flamelet surface-to-volume ratio in turbulent premixed combustion. Twenty-third Symposium (International) on Combustion 23(1), 809-815 (1991). DOI 10.1016/S0082-0784(06)80334-6

19. Catlin, C., Lindstedt, R.: Premixed turbulent burning velocities derived from mixing controlled reaction models with cold front quenching. Combustion and Flame 85(3-4), 427-439 (1991). DOI 10.1016/0010-2180(91)90145-2

20. Chakraborty, N., Champion, M., Mura, A., Swaminathan, N.: Modelling methods, Scalar dissipation rate approach. In: N. Swaminathan, K.N.C. Bray (eds.) Turbulent Premixed Flames, pp. 74-102. Cambridge University Press (2011)

21. Chakraborty, N., Rogerson, J.W., Swaminathan, N.: A priori assessment of closures for scalar dissipation rate transport in turbulent premixed flames using direct numerical sim- 
ulation. Physics of Fluids 20(4), 045,106 (2008). DOI 10.1063/1.2903846

22. Chen, Y.C., Peters, N., Schneemann, G.a., Wruck, N., Renz, U., Mansour, M.S.: The detailed flame structure of highly stretched turbulent premixed methane-air flames. Combustion and Flame 107(3), 223-244 (1996). DOI 10.1016/S0010-2180(96)00070-3

23. De, A., Acharya, S.: Large Eddy Simulation of a premixed Bunsen flame using a modified thickened-flame model at Two Reynolds number. Combustion Science and Technology 181(10), 1231-1272 (2009). DOI 10.1080/00102200903076266

24. Dodoulas, I.A., Navarro-Martinez, S.: Large Eddy Simulation of premixed turbulent flames using the probability density function approach. Flow, Turbulence and Combustion 90(3), 645-678 (2013). DOI 10.1007/s10494-013-9446-z

25. Dong, H.Q., Robin, V., Mura, A., Champion, M.: Analysis of Algebraic Closures of the Mean Scalar Dissipation Rate of the Progress Variable Applied to Stagnating Turbulent Flames. Flow, Turbulence and Combustion 90(2), 301-323 (2012). DOI 10.1007/s10494$012-9432-\mathrm{x}$

26. Furbo, E.: Evaluation of RANS turbulence models for flow problems with significant impact of boundary layers. Master's thesis, Uppsala Universitet (2010)

27. Fureby, C.: Homogenization based les for turbulent combustion. Flow, Turbulence and Combustion 84(3), 459-480 (2010). DOI 10.1007/s10494-009-9219-x

28. de Goey, L.P.H., Ten Thije Boonkkamp, J.H.M.: A flamelet description of premixed laminar flames and the relation with flame stretch. Combustion and Flame 119(3), 253-271 (1999). DOI 10.1016/S0010-2180(99)00052-8

29. Goodwin, D.G., Moffat, H.K., Speth, R.L.: Cantera: An object-oriented software toolkit for chemical kinetics, thermodynamics, and transport processes. http://www.cantera.org (2016). Version 2.2.1

30. Han, X., Morgans, A.S.: Simulation of the flame describing function of a turbulent premixed flame using an open-source LES solver. Combustion and Flame 162(5), 1778-1792 (2015). DOI 10.1016/j.combustflame.2014.11.039

31. Haworth, D.: Progress in probability density function methods for turbulent reacting flows. Progress in Energy and Combustion Science 36(2), 168-259 (2010). DOI 10.1016/j.pecs.2009.09.003 
32. Herrmann, M.: Numerical simulation of turbulent Bunsen flames with a level set flamelet model. Combustion and Flame 145(1-2), 357-375 (2006). DOI 10.1016/j.combustflame.2005.09.016

33. Iacovides, H., Launder, B., West, A.: A comparison and assessment of approaches for modelling flow over in-line tube banks. International Journal of Heat and Fluid Flow 49(C), 69-79 (2014). DOI 10.1016/j.ijheatfluidflow.2014.05.011

34. Jarrin, N., Benhamadouche, S., Laurence, D.R., Prosser, R.: A synthetic-eddy-method for generating inflow conditions for large-eddy simulations. International Journal of Heat and Fluid Flow 27, 585-593 (2006). DOI 10.1016/j.ijheatfluidflow.2006.02.006

35. Jarrin, N., Prosser, R., Uribe, J.C., Benhamadouche, S., Laurence, D.R.: Reconstruction of turbulent fluctuations for hybrid RANS/LES simulations using a Synthetic-Eddy Method. International Journal of Heat and Fluid Flow 30(3), 435-442 (2009). DOI 10.1016/j.ijheatfluidflow.2009.02.016

36. Jones, W.P., Launder, B.: The prediction of laminarization with a two-equation model of turbulence. International Journal of Heat and Mass Transfer 15(2), 301-314 (1972). DOI 10.1016/0017-9310(72)90076-2

37. Kha, K.Q.N., Losier, C., Robin, V., Mura, A., Champion, M.: Relevance of two basic turbulent premixed combustion models for the numerical simulations of V-shaped flames. Combustion Science and Technology 188(11-12), 1878-1903 (2016). DOI $10.1080 / 00102202.2016 .1211866$

38. Klein, T., Craft, T.J., Iacovides, H.: Assessment of the performance of different classes of turbulence models in a wide range of non-equilibrium flows. International Journal of Heat and Fluid Flow 51, 229-256 (2015). DOI 10.1016/j.ijheatfluidflow.2014.10.017

39. Klimenko, A., Bilger, R.W.: Conditional moment closure for turbulent combustion. Progress in Energy and Combustion Science 25(6), 595-687 (1999). DOI 10.1016/S03601285(99)00006-4

40. Kolla, H., Rogerson, J.W., Chakraborty, N., Swaminathan, N.: Scalar dissipation rate modeling and its validation. Combustion Science and Technology 181(3), 518-535 (2009). DOI 10.1080/00102200802612419

41. Kolla, H., Swaminathan, N.: Strained flamelets for turbulent premixed flames, I: Formulation and planar flame results. Combustion and Flame 157(5), 943-954 (2010). DOI 
10.1016/j.combustflame.2010.01.018

42. Kolla, H., Swaminathan, N.: Strained flamelets for turbulent premixed flames II: Laboratory flame results. Combustion and Flame 157(7), 1274-1289 (2010). DOI 10.1016/j.combustflame.2010.03.016

43. Kronenburg, A., Mastorakos, E.: The Conditional Moment Closure Model. In: T. Mastorakos E.and Echekki (ed.) Turbulent Combustion Modeling, pp. 63-90. Springer (2011)

44. Kuan, T.S., Lindstedt, R.P., Vaos, E.M.: Higher moment based modeling of turbulence enhanced explosion kernels in confined fuel-air mixtures. In: G.D. Roy (ed.) Advances in Confined Detonations and Pulse Detonation Engines, pp. 17-40. Torus Press, Moscow (2003)

45. Langella, I., Swaminathan, N., Gao, Y., Chakraborty, N.: Assessment of dynamic closure for premixed combustion large eddy simulation. Combustion Theory and Modelling 19(5), 628-656 (2015). DOI 10.1080/13647830.2015.1080387

46. Libby, P.A., Bray, K.N.C.: Countergradient diffusion in premixed turbulent flames. AIAA Journal 19(2), 205-213 (1981). DOI 10.2514/3.50941

47. Lindstedt, R., Vaos, E.: Transported PDF modeling of high-Reynolds-number premixed turbulent flames. Combustion and Flame 145(3), 495-511 (2006). DOI 10.1016/j.combustflame.2005.12.015

48. Mantel, T., Borghi, R.: A new model of premixed wrinkled flame propagation based on a scalar dissipation equation. Combustion and Flame 96(4), 443-457 (1994). DOI 10.1016/0010-2180(94)90110-4

49. Martin, S.M., Kramlich, J.C., Kosaly, G., Riley, J.J.: The Premixed Conditional Moment Closure Method applied to idealized lean premixed gas turbine combustors. Journal of Engineering for Gas Turbines and Power 125(October 2003), 573-580 (2002). DOI 10.1115/GT2002-30094

50. Mura, A., Borghi, R.: Towards an extended scalar dissipation equation for turbulent premixed combustion. Combustion and Flame 133(1-2), 193-196 (2003). DOI 10.1016/S00102180(02)00565-5

51. Navarro-Martinez, S., Kronenburg, A., Di Mare, F.: Conditional moment closure for large eddy simulations. Flow, Turbulence and Combustion 75(1-4), 245-274 (2005). DOI $10.1007 / \mathrm{s} 10494-005-8580-7$ 
52. Nikolaou, Z.M.: Study of multi-component fuel premixed combustion using direct numerical simulation. Ph.D. thesis, University Of Cambridge (2014)

53. Nikolaou, Z.M., Swaminathan, N.: Direct Numerical Simulation of complex fuel combustion with detailed chemistry: Physical insight and mean reaction rate modeling. Combustion Science and Technology 187(11), 1759-1789 (2015). DOI $10.1080 / 00102202.2015 .1064911$

54. Pitsch, H., Duchamp de Lageneste, L.: Large-eddy simulation of premixed turbulent combustion using a level-set approach. Proceedings of the Combustion Institute 29(2), 20012008 (2002). DOI 10.1016/S1540-7489(02)80244-9

55. Pitz, R.W., Daily, J.W.: An experimental study of combustion: The turbulent structure of a reacting shear layer formed at a rearward-facing step. Tech. rep., NASA, Lewis research center (contract number 165427) (1981)

56. Pitz, R.W., Daily, J.W.: Combustion in a turbulent mixing layer formed at a rearwardfacing step. AIAA Journal 21(11), 1565-1570 (1983). DOI 10.2514/3.8290

57. Poinsot, T.J., Veynante, D.: Theoretical and numerical combustion, 2nd edn. R.T.Edwards, Inc (2005)

58. Pope, S.B.: An explanation of the turbulent round-jet/plane-jet anomaly. AIAA Journal 16(3), 279-281 (1978). DOI 10.2514/3.7521

59. Pope, S.B.: PDF methods for turbulent reactive flows. Progress in Energy and Combustion Science 11(2), 119-192 (1985). DOI 10.1016/0360-1285(85)90002-4

60. Pope, S.B.: The evolution of surfaces in turbulence. International Journal of Engineering Science 26(5), 445-469 (1988). DOI 10.1016/0020-7225(88)90004-3

61. Prasad, R., Gore, J.: An evaluation of flame surface density models for turbulent premixed jet flames. Combustion and Flame 116(1-2), 1-14 (1999). DOI 10.1016/S00102180(98)00046-7

62. Salehi, M.M., Bushe, W.K.: Presumed PDF modeling for RANS simulation of turbulent premixed flames. Combustion Theory and Modelling 14(3), 381-403 (2010). DOI $10.1080 / 13647830.2010 .489957$

63. Salehi, M.M., Bushe, W.K., Daun, K.J.: Application of the conditional source-term estimation model for turbulence-chemistry interactions in a premixed flame. Combustion Theory and Modelling 16(2), 301-320 (2012). DOI 10.1080/13647830.2011.621029 
64. Sankaran, R., Hawkes, E.R., Yoo, C.S., Chen, J.H.: Response of flame thickness and propagation speed under intense turbulence in spatially developing lean premixed methaneair jet flames. Combustion and Flame 162(9), 3294-3306 (2015). DOI 10.1016/j.combustflame.2015.05.019

65. Smith, G.P., Golden, D.M., Frenklach, M., Moriarty, N.W., Eiteneer, B., Goldenberg, M., Bowman, C.T., Hanson, R.K., Song, S., Gardiner, W.C., Lissianski, J.V.V., Qin, Z.: GRI-MECH 3.0. http://www.me.berkeley.edu/gri_mech/

66. Spalding, D.B.: Mixing and chemical reaction in steady confined turbulent flames. Symposium (International) on Combustion 13(1), 649-657 (1971). DOI 10.1016/S00820784(71)80067-X

67. Swaminathan, N., Bray, K.N.C.: Effect of dilatation on scalar dissipation in turbulent premixed flames. Combustion and Flame 143(4), 549-565 (2005). DOI 10.1016/j.combustflame.2005.08.020

68. Tangermann, E., Keppeler, R., Pfitzner, M.: Premixed turbulent combustion models for large eddy and RANS simulations. In: ASME Turbo Expo 2010: Power for Land, Sea, and Air, pp. 203-212 (2010). DOI 10.1115/GT2010-22298

69. Versteeg, H.K., Malalasekera, W.: An Introduction to Computational Fluid Dynamics The Finite Volume Method, 2nd edn. Pearson Education Limited (2007)

70. Veynante, D., Trouvé, A., Bray, K.N.C., Mantel, T.: Gradient and counter-gradient scalar transport in turbulent premixed flames. Journal of Fluid Mechanics 332, 263-293 (1997). DOI $10.1017 /$ S0022112096004065

71. Weller, H.G., Tabor, G., Gosman, A., Fureby, C.: Application of a flame-wrinkling LES combustion model to a turbulent mixing layer. Symposium (International) on Combustion 27(1), 899-907 (1998). DOI 10.1016/S0082-0784(98)80487-6 


\begin{tabular}{|c|c|c|c|}
\hline Scalar dissipation models & $T_{1}$ & $T_{2}$ & $T_{3}$ \\
\hline SDR-1 & $2 K_{c}^{*} \frac{u_{L}^{0}}{\delta_{L}^{0}}$ & $C_{3} \frac{\widetilde{\epsilon}}{\widetilde{k}}-\left(\tau C_{4} D a_{L}\right) \frac{\widetilde{\epsilon}}{\widetilde{k}}$ & $\frac{c^{\prime \prime 2}}{\beta^{\prime}}$ \\
\hline SDR-2 & $C_{D_{c}} \frac{u_{L}^{0}}{\delta_{L}^{0}}$ & $C_{D} \frac{\widetilde{\epsilon}}{\widetilde{k}}$ & $\left(1+\frac{2}{3} C_{\epsilon_{c}} \frac{u_{L}^{0}}{\sqrt{\widetilde{k}}}\right) \widetilde{c^{\prime \prime 2}}$ \\
\hline SDR-3 & N/A & $C_{D} \frac{\widetilde{\epsilon}}{\widetilde{k}}$ & $\left(1+\frac{2}{3} C_{\epsilon_{c}} \frac{u_{L}^{0}}{\sqrt{\widetilde{k}}}\right) \widetilde{c^{\prime \prime 2}}$ \\
\hline SDR-4 & N/A & $\frac{C_{\phi}}{4} \frac{\widetilde{\epsilon}}{\widetilde{k}}$ & $\left(1+C_{\phi}^{*}(1+\tau \widetilde{c}) u_{L}^{0} \frac{R e_{t}^{1 / 4}}{\sqrt{\widetilde{k}}}\right) \widetilde{c^{\prime \prime 2}}$ \\
\hline
\end{tabular}

Table 1 Algebraic scalar dissipation models. All the quantities in this table are defined in sections 2.1.1-2.1.4

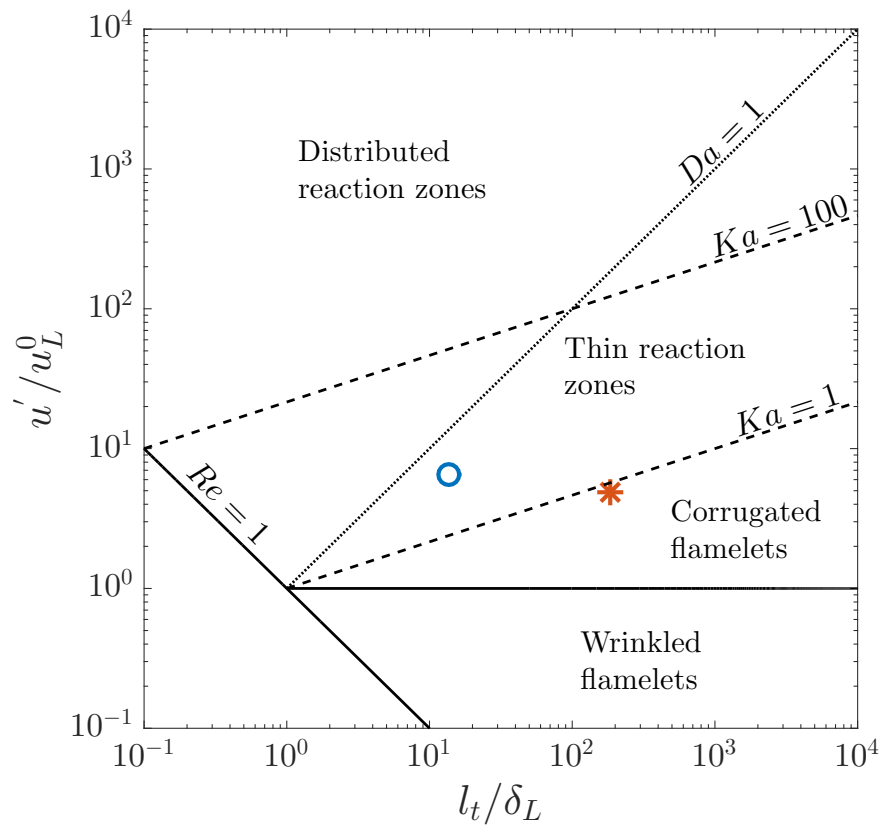

Fig. 1 The regime diagram with the parameters of the dump combustor (*) and Bunsen flame

(o)

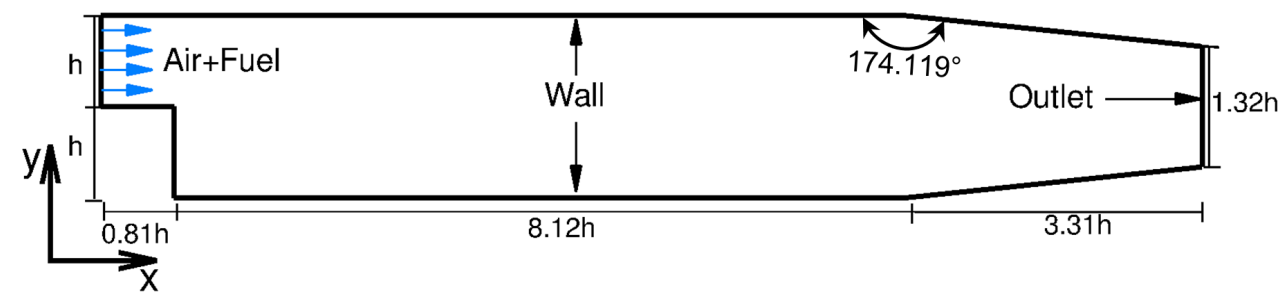

Fig. 2 Schematic of the computational domain $(h=0.0254 m)$. 


\begin{tabular}{|c|c|c|c|c|c|}
\hline$u_{0}(\mathrm{~m} / \mathrm{s})$ & $p_{0}(\mathrm{~atm})$ & $T_{0}(\mathrm{~K})$ & $u_{L}^{0}(\mathrm{~m} / \mathrm{s})$ & $\delta_{L}^{0}(\mathrm{~mm})$ & $\nu_{u} \mathrm{~m}^{2} / \mathrm{s}$ \\
\hline 13.3 & 1 & 293 & 0.09 & 1.6 & $2.183 \times 10^{-5}$ \\
\hline
\end{tabular}

Table 2 Inlet parameters for the dump combustor simulation.

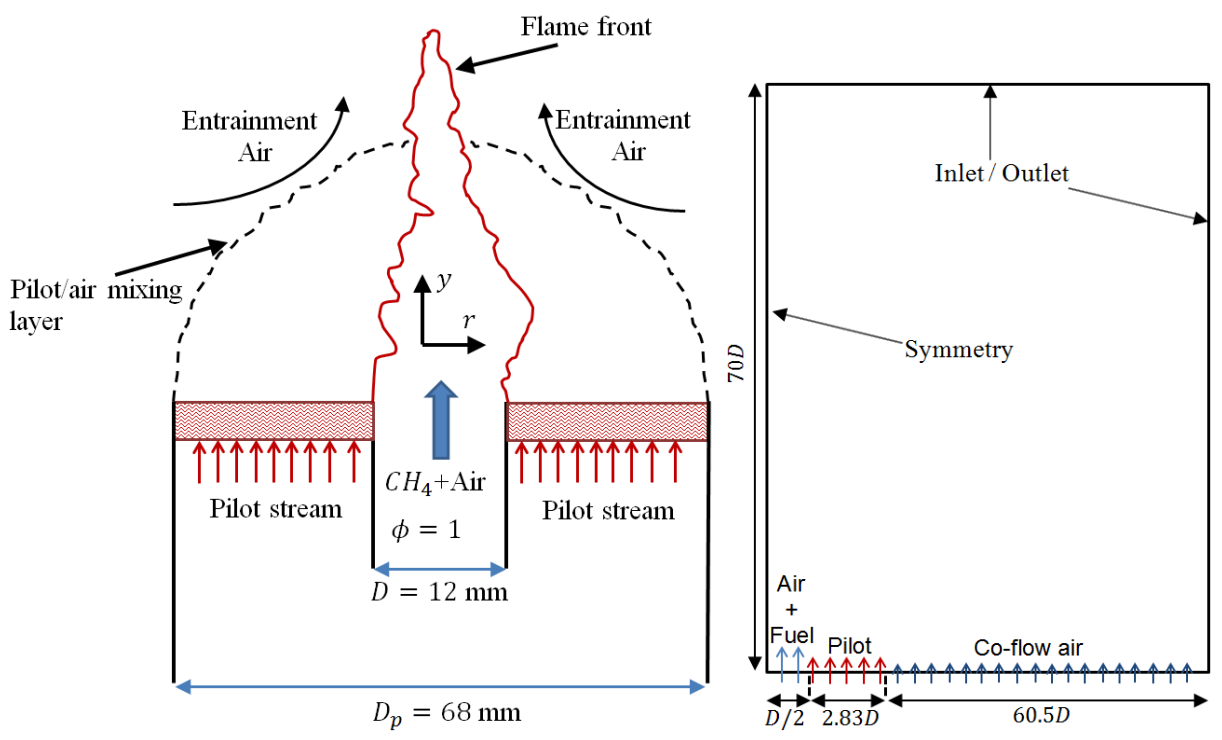

(a) Experimental configuration for the Bunsen flame (b) Schematic of the simulated domain

Fig. 3 Bunsen flame flow configuration

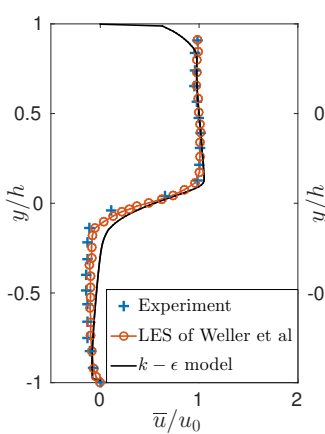

(a) $x / h=1$

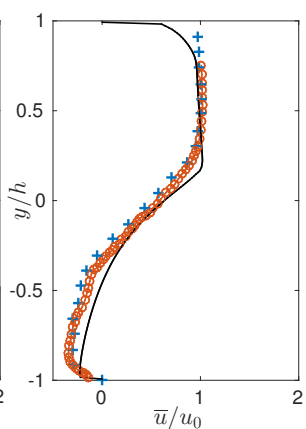

(b) $x / h=3$

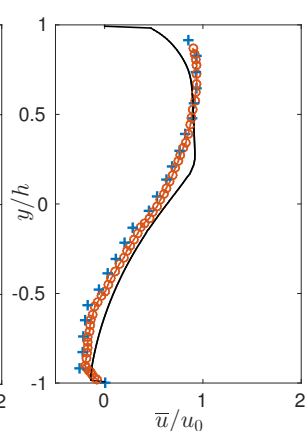

(c) $x / h=5$

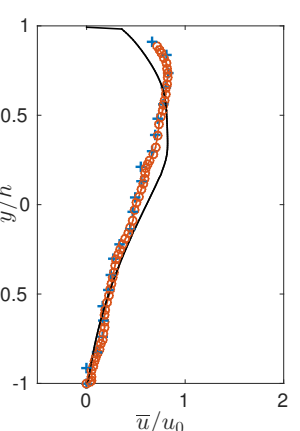

(d) $x / h=7$

Fig. 4 Isothermal flow velocity profiles for the dump combustor case. 


\begin{tabular}{|c|c|}
\hline Model name & Test parameters \\
\hline SDR-1.1 & $\beta^{\prime}=6.7$ with transport equation for $\widehat{c^{\prime \prime} 2}$ \\
\hline SDR-1.2 & $\beta^{\prime}=8$ with transport equation for $\widehat{c^{\prime \prime 2}}$ \\
\hline SDR-1.3 & $\beta^{\prime}=20$ with transport equation for $\widetilde{c^{\prime \prime 2}}$ \\
\hline SDR-1.4 & $\beta^{\prime}=40$ with transport equation for $\widetilde{c^{\prime \prime 2}}$ \\
\hline SDR-1.5 & $\beta^{\prime}=6.7$ with BML closure in Eq. 2 \\
\hline SDR-1.6 & $\beta^{\prime}=8$ with BML closure in Eq. 2 \\
\hline SDR-1.7 & $\beta^{\prime}=20$ with BML closure in Eq. 2 \\
\hline SDR-1.8 & $\beta^{\prime}=40$ with BML closure in Eq. 2 \\
\hline SDR-2.1 & $C_{D}=0.1$ \\
\hline SDR-2.2 & $C_{D}=0.25$ \\
\hline SDR-2.3 & $C_{D}=0.5$ \\
\hline SDR-3.1 & $C_{D}=0.25$ \\
\hline SDR-3.2 & $C_{D}=0.5$ \\
\hline SDR-3.3 & $C_{D}=1.0$ \\
\hline SDR-4.1 & $C_{\phi}=4.0$ \\
\hline SDR-4.2 & $C_{\phi}=2.0$ \\
\hline
\end{tabular}

Table 3 Different variations of scalar dissipation models.

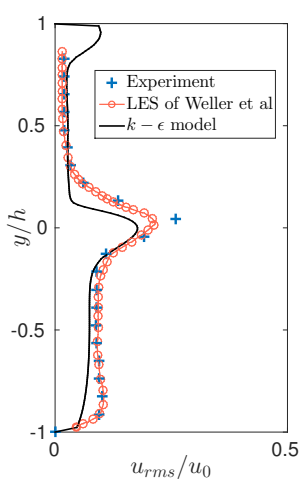

(a) $x / h=1$

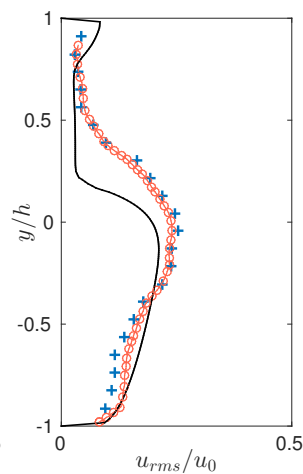

(b) $x / h=3$

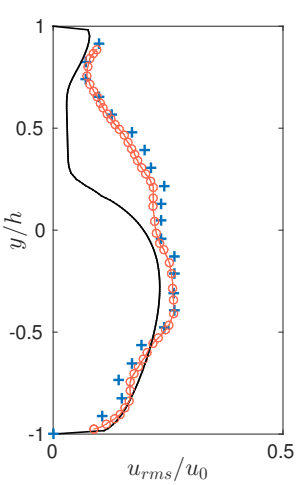

(c) $x / h=5$

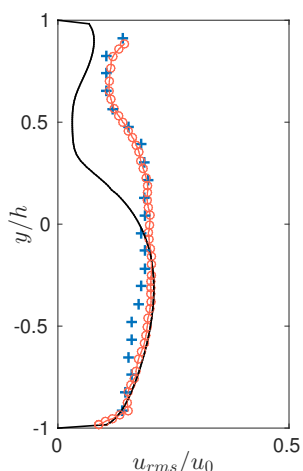

(d) $x / h=7$

Fig. 5 Isothermal flow root mean squared velocity profiles for the dump combustor case. 


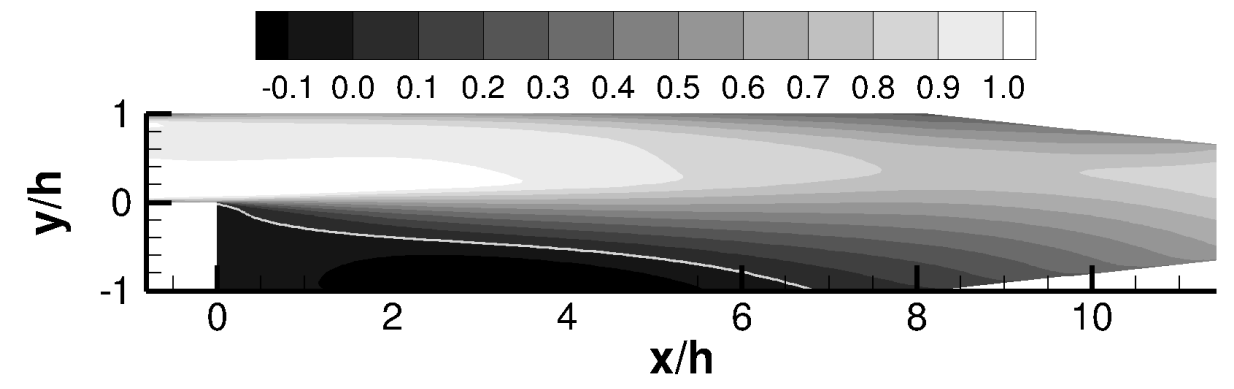

Fig. 6 Isothermal flow velocity $\left(\bar{u} / u_{0}\right)$. The white line represents $\bar{u} / u_{0}=0$, which indicates the recirculation region.

\begin{tabular}{|c|c|}
\hline Case & Length of the recirculation zone $\left(x_{r} / h\right)$ \\
\hline Experiment & 4.50 \\
\hline SDR-1.1 & 4.24 \\
\hline SDR-1.2 & 4.50 \\
\hline SDR-1.5 & 4.24 \\
\hline SDR-1.6 & 4.50 \\
\hline SDR-2.1 & 4.40 \\
\hline SDR-2.2 & 4.13 \\
\hline SDR-3.1 & 5.51 \\
\hline SDR-3.2 & 5.12 \\
\hline SDR-3.3 & 4.15 \\
\hline SDR-4.1 & 4.20 \\
\hline
\end{tabular}

Table 4 Length of the recirculation zone predicted by different models.

\begin{tabular}{|c|c|c|}
\hline$x / h$ & maximum \% difference in $C_{3}$ & maximum $\%$ difference in $C_{4}$ \\
\hline 1 & 3.3 & 11.3 \\
\hline 3 & 4.6 & 11.9 \\
\hline 5 & 7.4 & 14.1 \\
\hline 7 & 11.3 & 21.3 \\
\hline
\end{tabular}

Table 5 Percentage difference in the values of the scaling factors $C_{3}$ and $C_{4}$ with change in $\beta^{\prime}=6.7(\mathrm{SDR}-1.1)$ to $\beta^{\prime}=8.0(\mathrm{SDR}-1.2)$. 


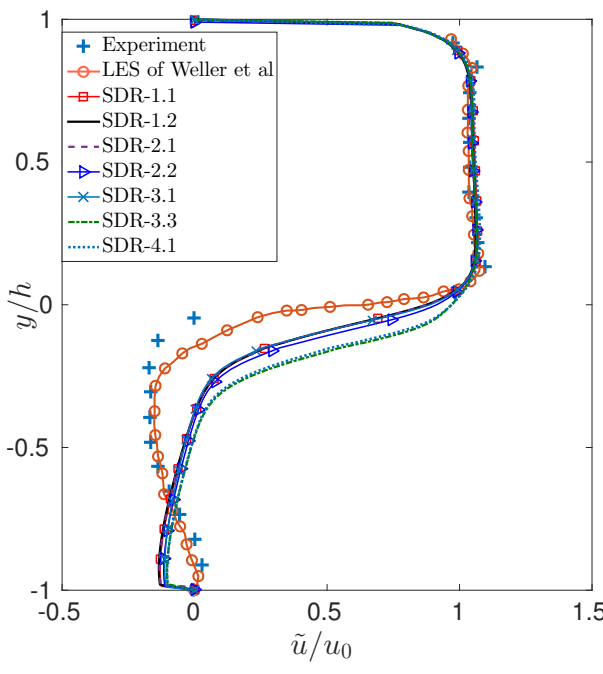

(a) $x / h=1$

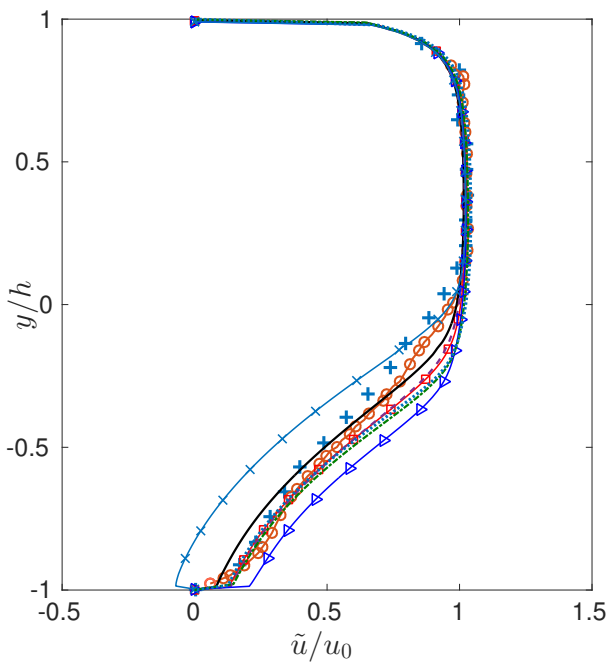

(c) $x / h=5$

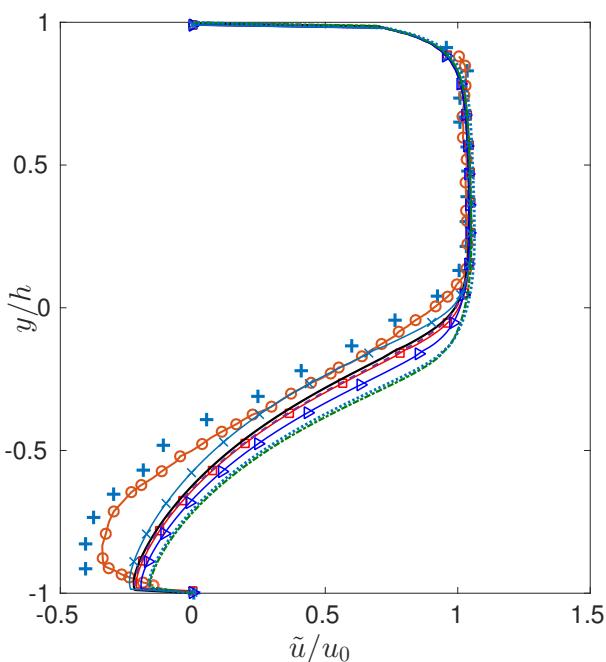

(b) $x / h=3$

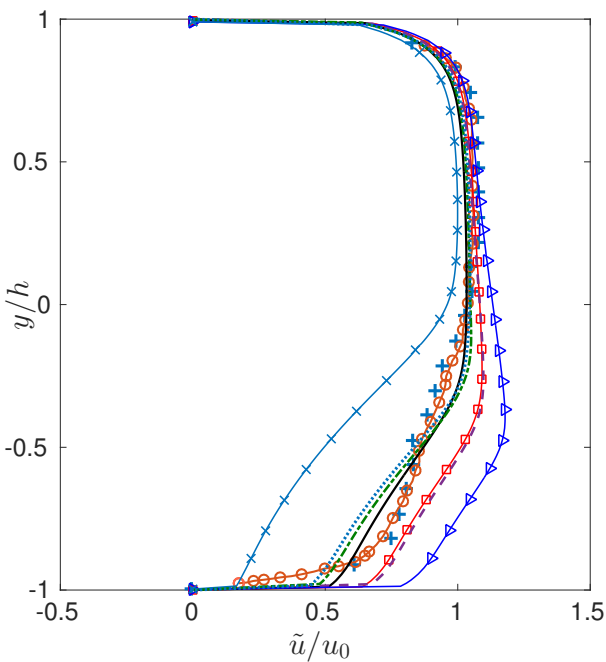

(d) $x / h=7$

Fig. 7 Reacting flow velocity predicted by different models for the dump combustor case. 


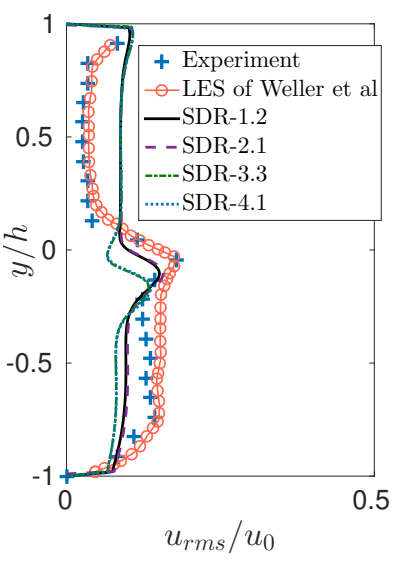

(a) $x / h=1$

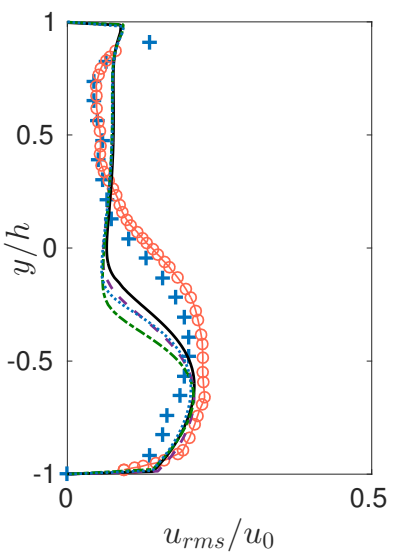

(c) $x / h=5$

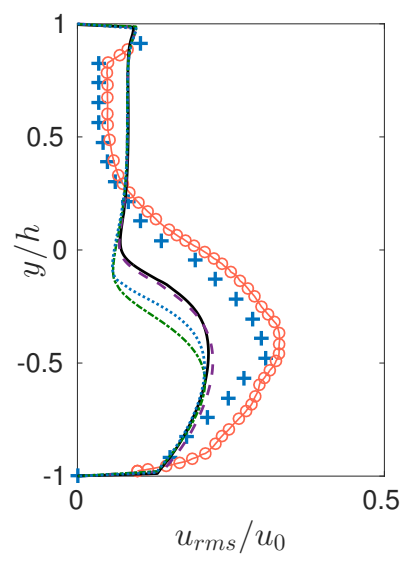

(b) $x / h=3$

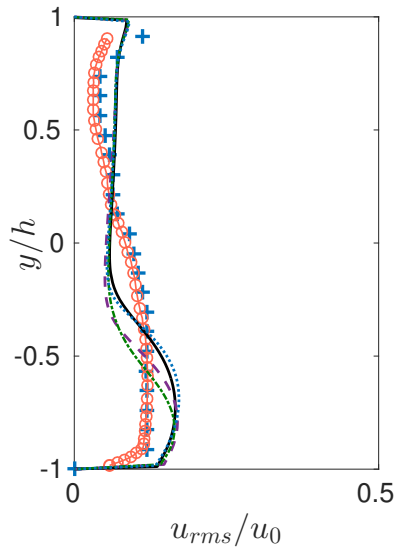

(d) $x / h=7$

Fig. 8 Reacting flow root mean squared velocity predicted by different models for the dump combustor case.

\begin{tabular}{|c|c|c|}
\hline$y / D$ & maximum $\%$ difference in $C_{3}$ & maximum $\%$ difference in $C_{4}$ \\
\hline 2.5 & 187.8 & 82.2 \\
\hline 4.5 & 189.7 & 78.0 \\
\hline 6.5 & 187.7 & 59.8 \\
\hline 8.5 & 152.8 & 37.2 \\
\hline
\end{tabular}

Table 6 Percentage difference in the values of the scaling factors $C_{3}$ and $C_{4}$ with change in $\beta^{\prime}=6.7\left(\mathrm{SDR}-1.1\right.$ model) to $\beta^{\prime}=40.0$ (SDR-1.4 model). 


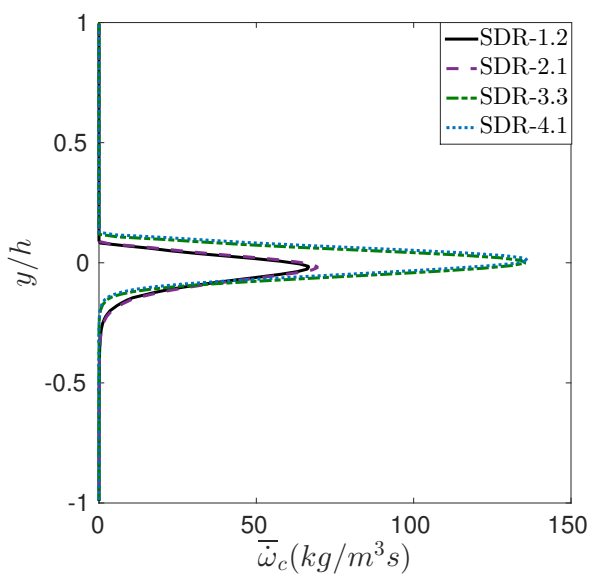

(a) $x / h=1$

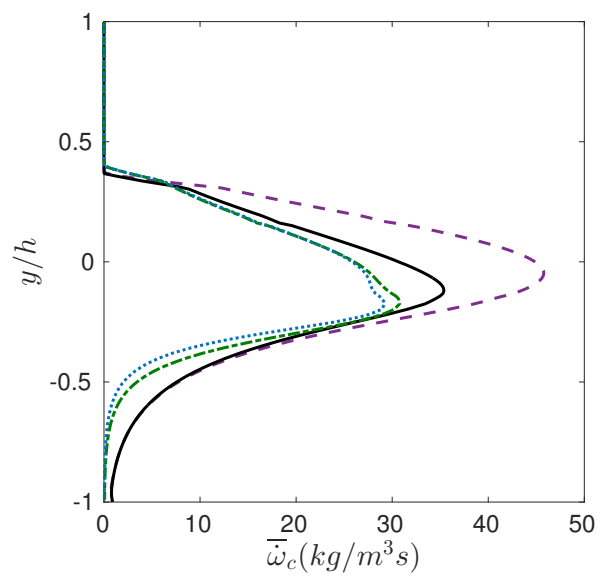

(c) $x / h=5$

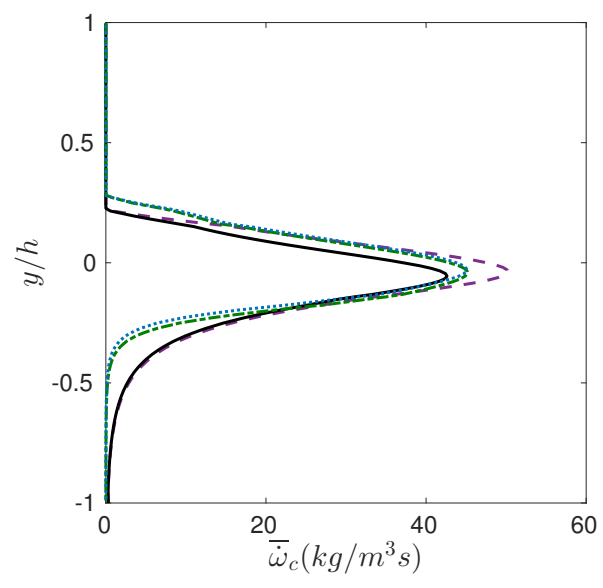

(b) $x / h=3$

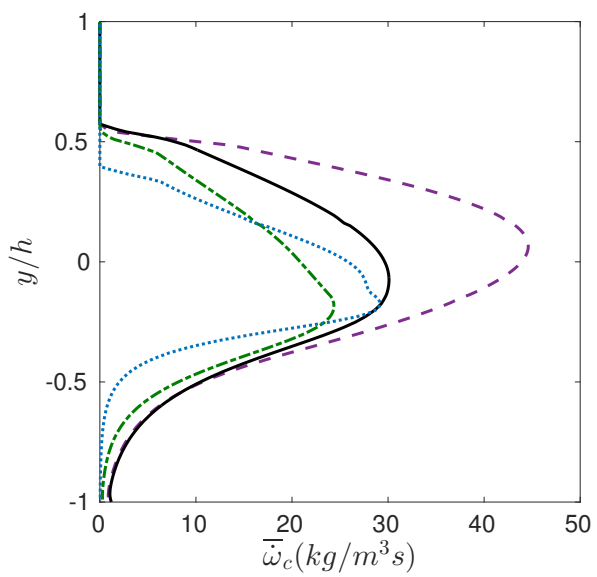

(d) $x / h=7$

Fig. 9 Reaction rate predicted by different models for the dump combustor case.

\begin{tabular}{|c|c|c|}
\hline Models & Wrinkled/Corrugated flamelets & Thin reaction zone \\
\hline SDR-1 & $\beta^{\prime}=8$ & $\beta^{\prime}=40$ with $\widetilde{c^{\prime \prime 2}}$ transport equation \\
\hline SDR-2 & $C_{D}=0.1$ & $C_{D}=0.1$ \\
\hline SDR-3 & $C_{D}=1$ & $C_{D}=0.5$ \\
\hline SDR-4 & $C_{\phi}=4$ & $C_{\phi}=2$ \\
\hline
\end{tabular}

Table 7 Recommended constants for different algebraic scalar dissipation models to be used in different combustion regimes. 


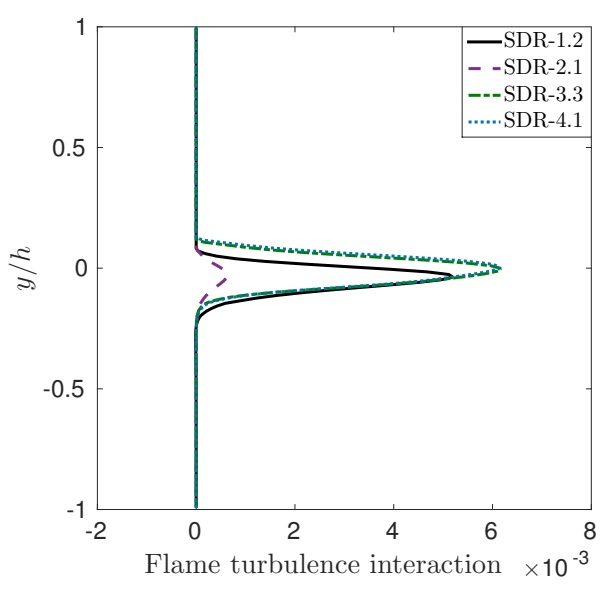

(a) $x / h=1$

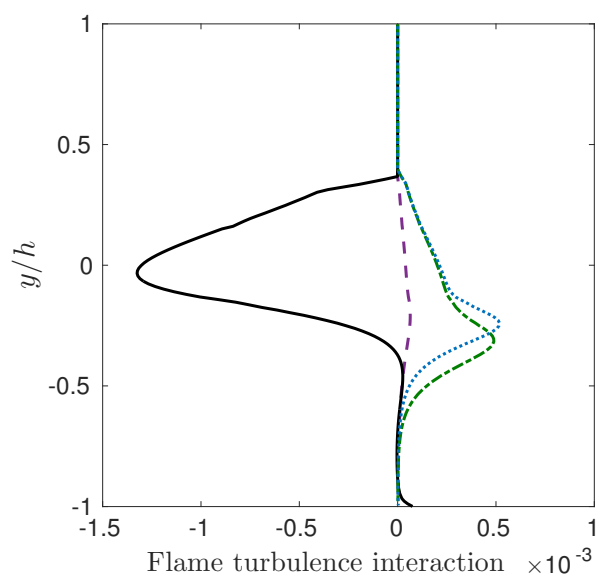

(c) $x / h=5$

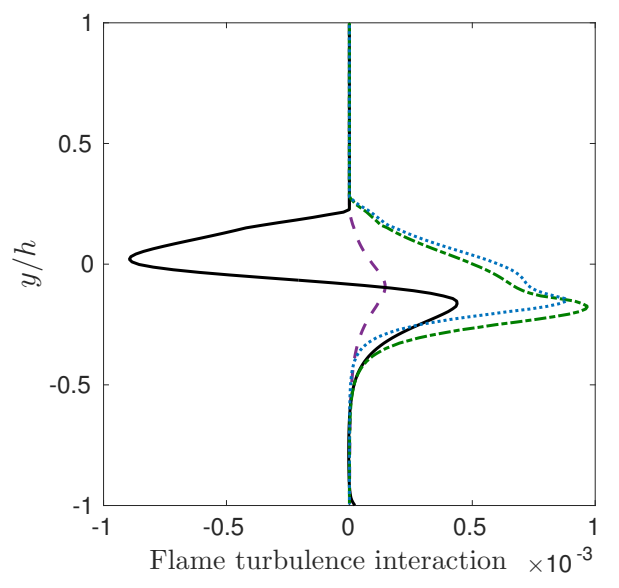

(b) $x / h=3$

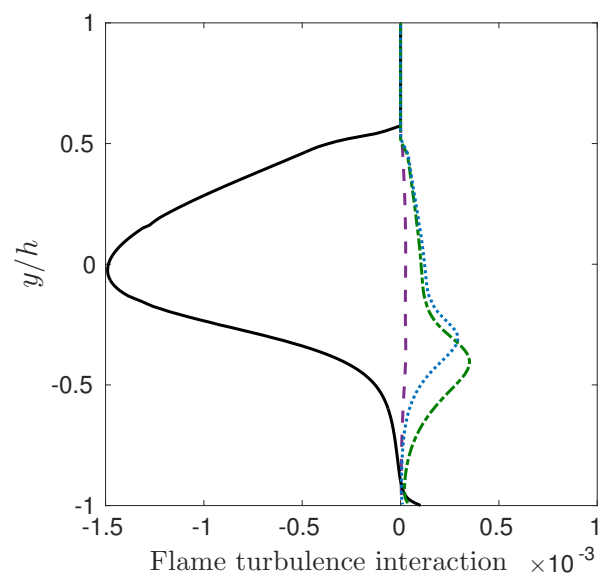

(d) $x / h=7$

Fig. 10 Flame turbulence interaction $\left(\overline{\rho \alpha \nabla c^{\prime \prime} . \mathbf{S}^{\prime \prime} \cdot \nabla \mathbf{c}^{\prime \prime}}\right)$ predicted by different models for the dump combustor case. The values are normalised using the respective $\rho_{u}, \delta_{L}^{0}$ and $u_{L}^{0}$. 


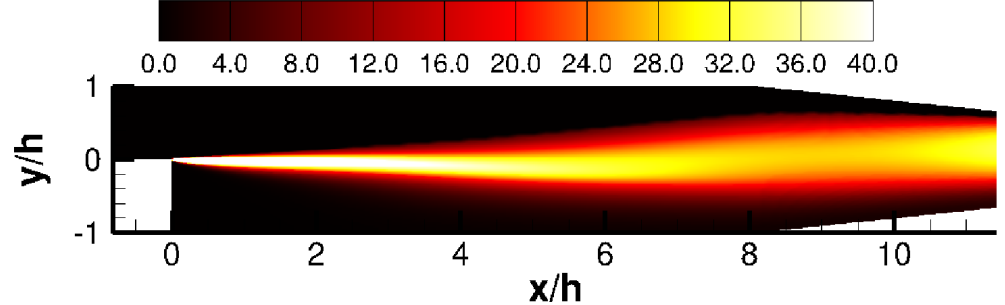

(a) reaction rate $\overline{\dot{\omega}}_{c}\left(\mathrm{~kg} / \mathrm{m}^{3} \mathrm{~s}\right)$ prediction by using SDR-1.2 model.

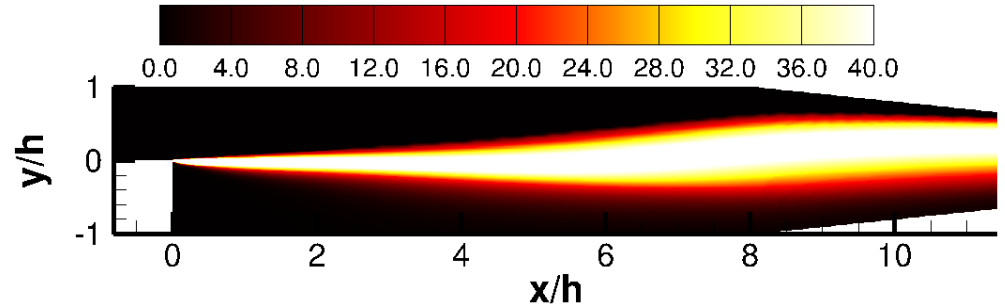

(b) reaction rate $\overline{\dot{\omega}}_{c}\left(\mathrm{~kg} / \mathrm{m}^{3} \mathrm{~s}\right)$ prediction by using SDR-2.1 model.

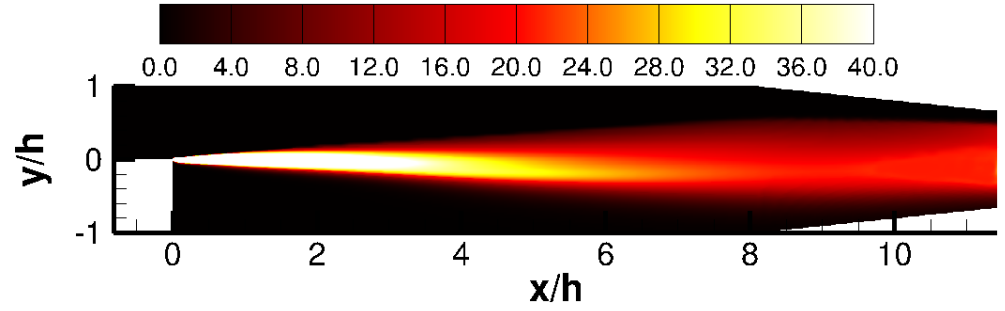

(c) reaction rate $\overline{\dot{\omega}}_{c}\left(\mathrm{~kg} / \mathrm{m}^{3} \mathrm{~s}\right)$ prediction by using SDR-3.3 model.

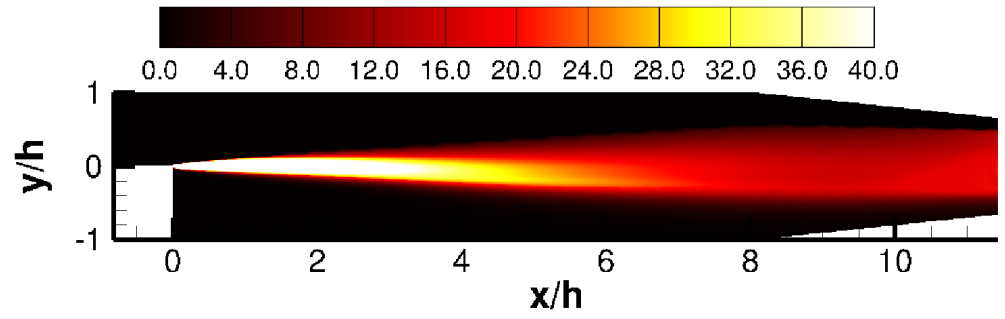

(d) reaction rate $\overline{\dot{\omega}}_{c}\left(\mathrm{~kg} / \mathrm{m}^{3} \mathrm{~s}\right)$ prediction by using SDR-4.1 model.

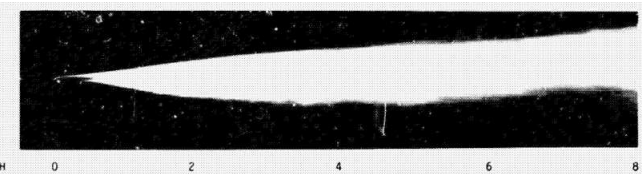

(e) Schileren photograph of the flame with an expo-

sure time of $33 m s$ (from [55])

Fig. 11 Flame location and reaction rate for the dump combustor case. 


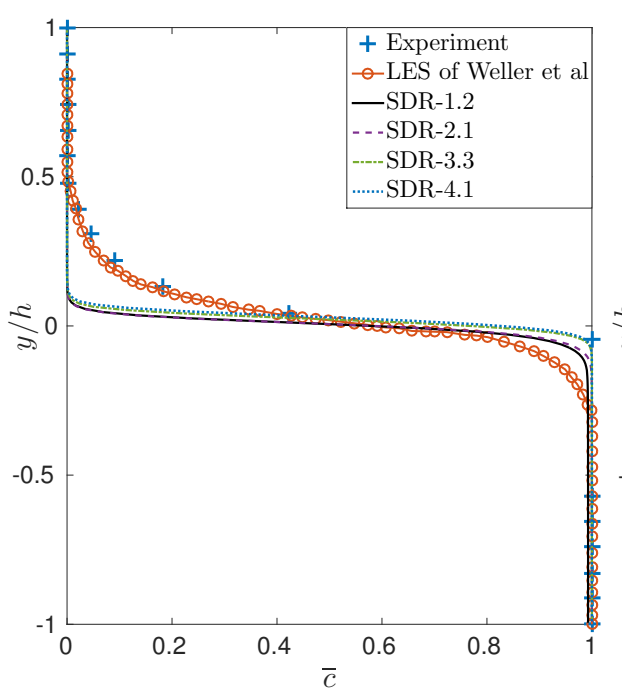

(a) $x / h=0.4$

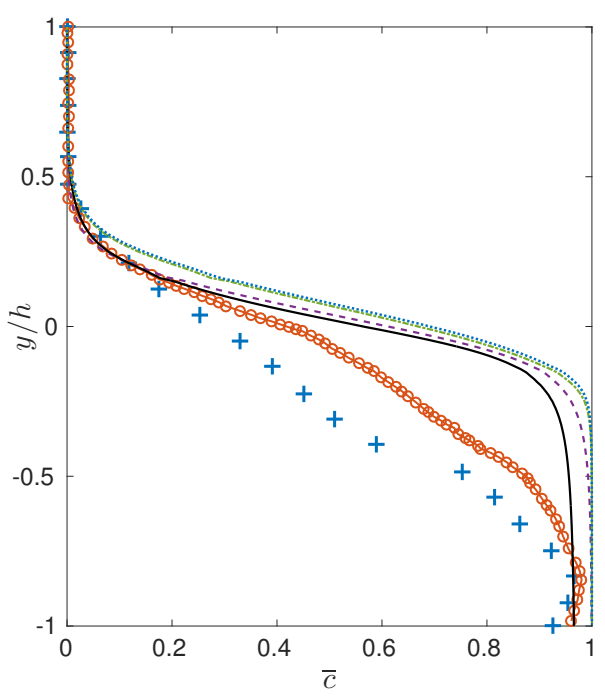

(c) $x / h=3.5$

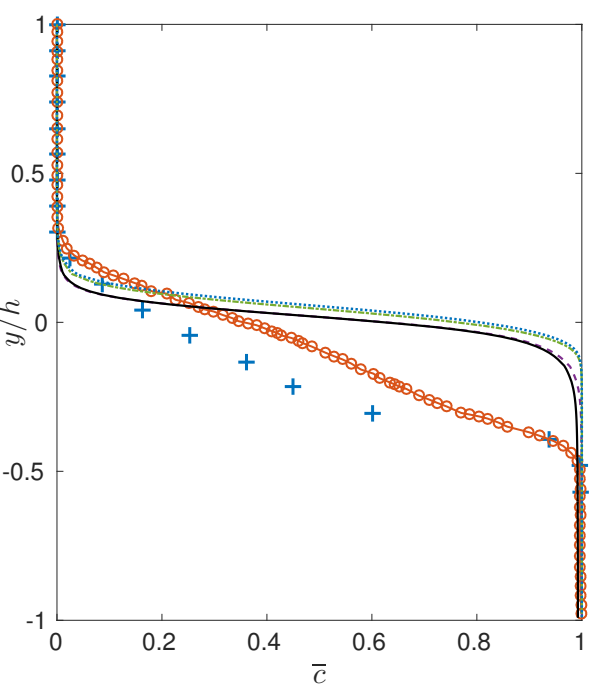

(b) $x / h=1.2$

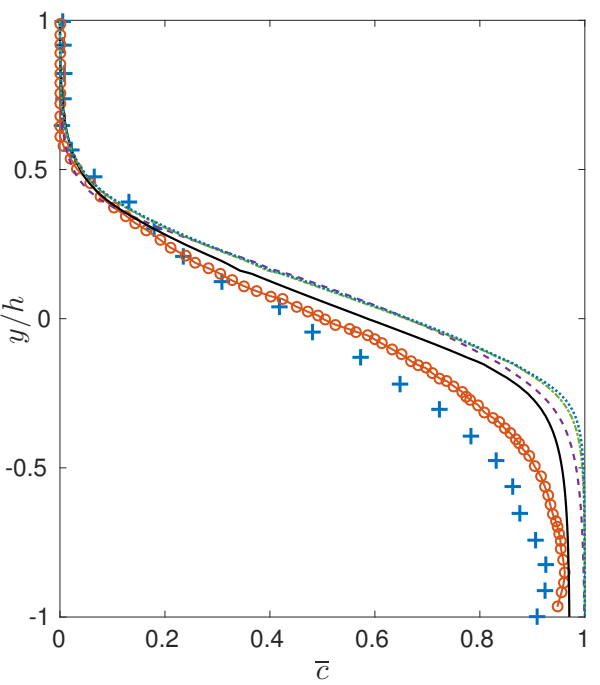

(d) $x / h=5.4$

Fig. 12 Reacting flow progress variable predicted by different models for the dump combustor case. 

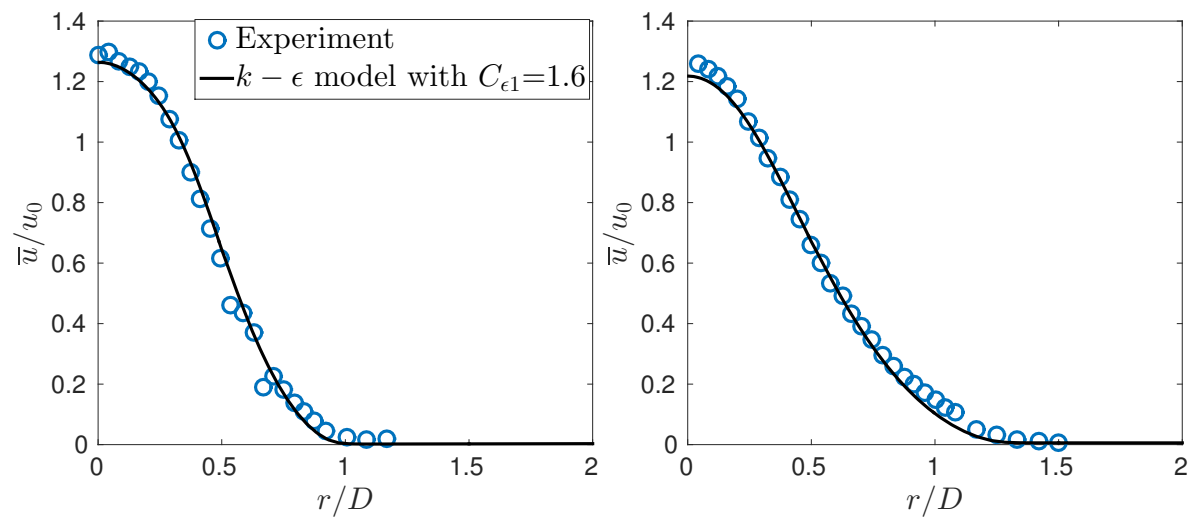

(a) $y / D \approx 2.5$

(b) $y / D \approx 4.5$

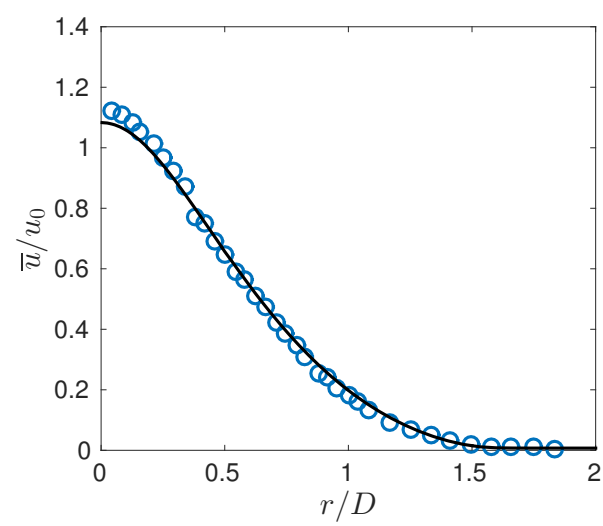

(c) $y / D \approx 6.5$

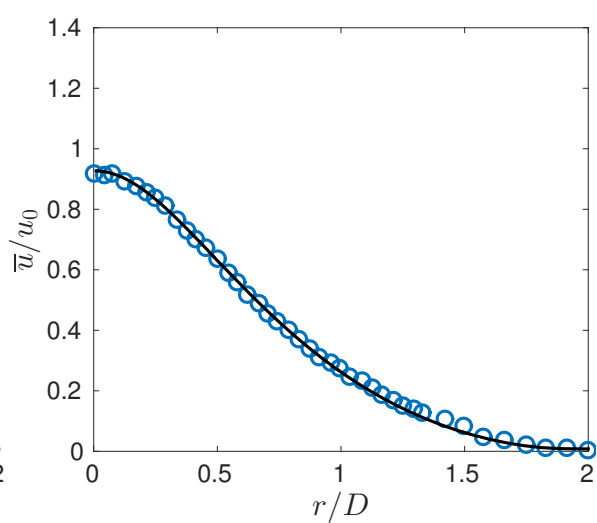

(d) $y / D \approx 8.5$

Fig. 13 Isothermal flow velocity profiles for the Bunsen flame calculation. 


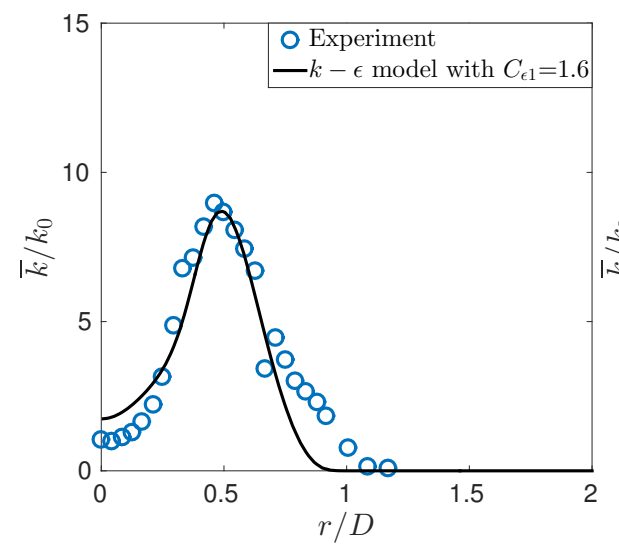

(a) $y / D \approx 2.5$

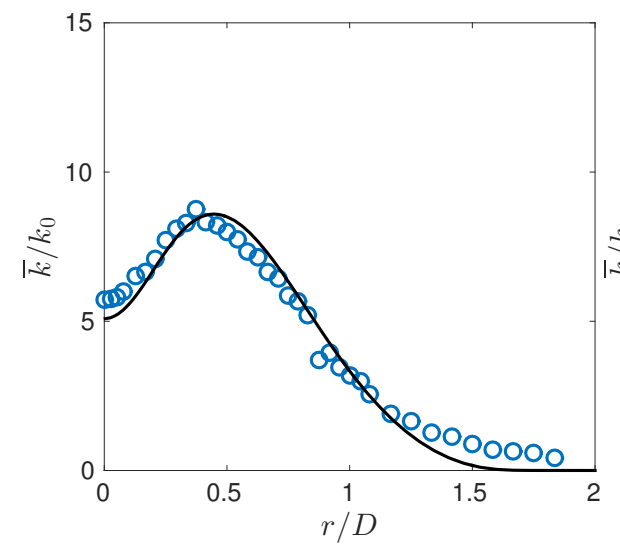

(c) $y / D \approx 6.5$

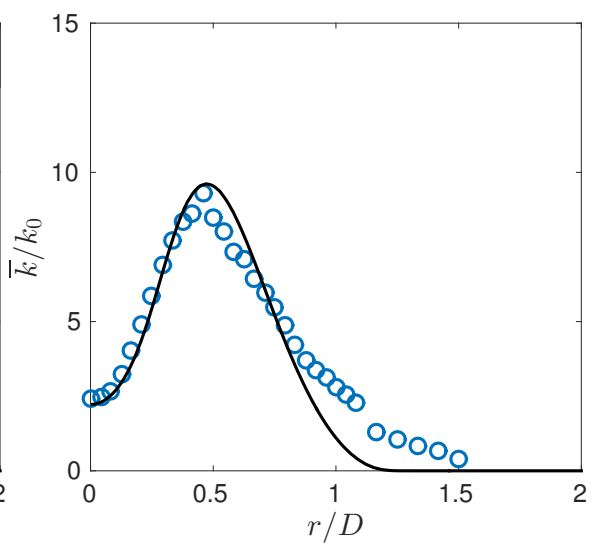

(b) $y / D \approx 4.5$

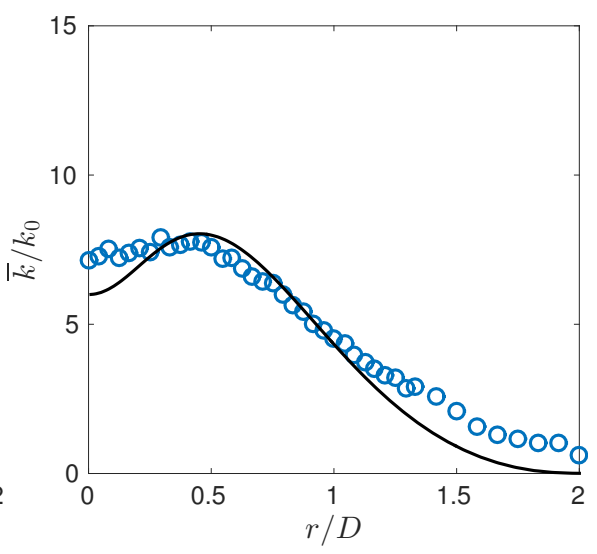

(d) $y / D \approx 8.5$

Fig. 14 Isothermal flow turbulent kinetic energy profiles for the Bunsen flame calculation. 


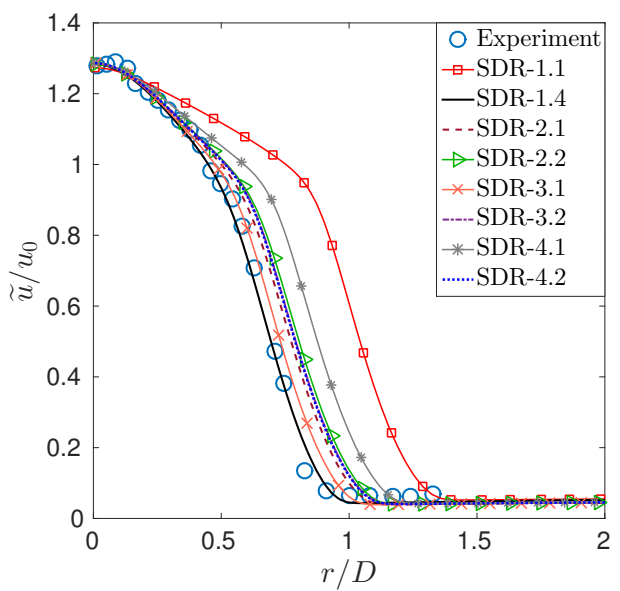

(a) $y / D \approx 2.5$

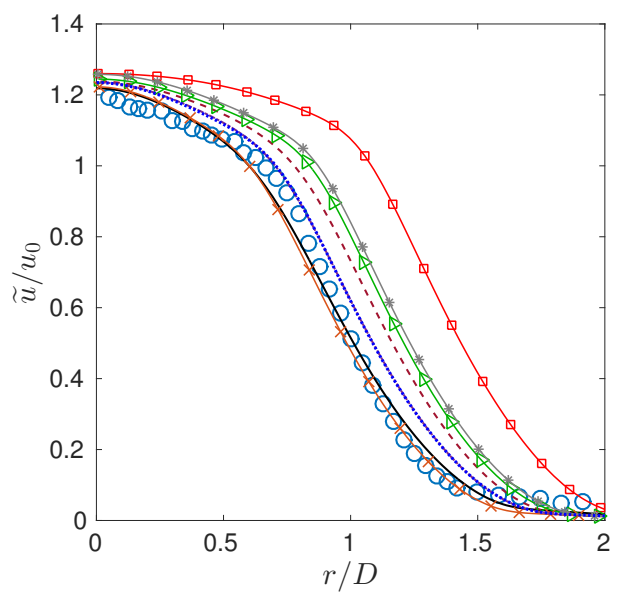

(c) $y / D \approx 6.5$

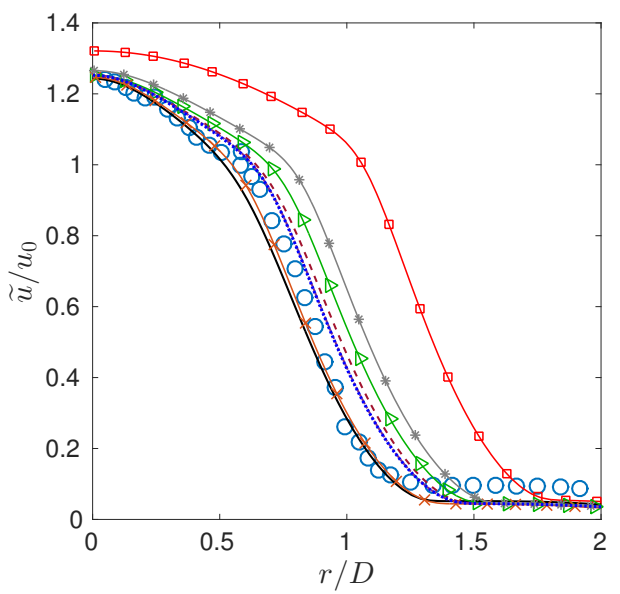

(b) $y / D \approx 4.5$

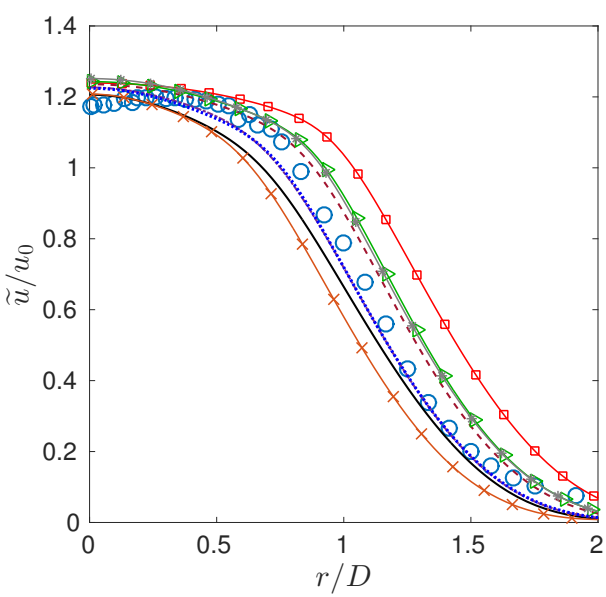

(d) $y / D \approx 8.5$

Fig. 15 Reacting flow velocity profiles for the Bunsen flame calculation. 


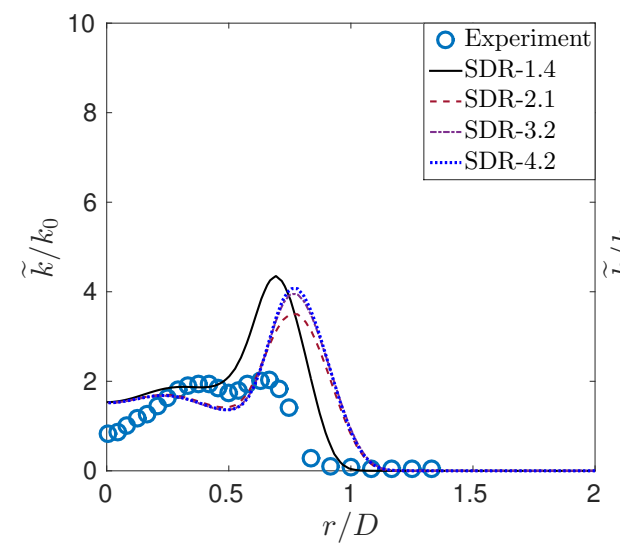

(a) $y / D \approx 2.5$

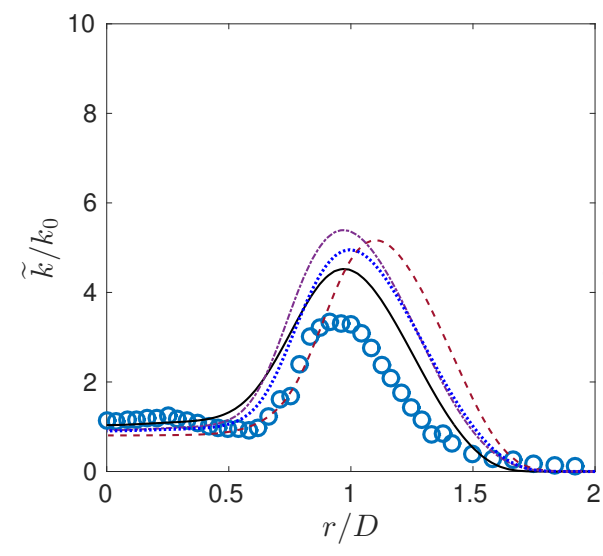

(c) $y / D \approx 6.5$

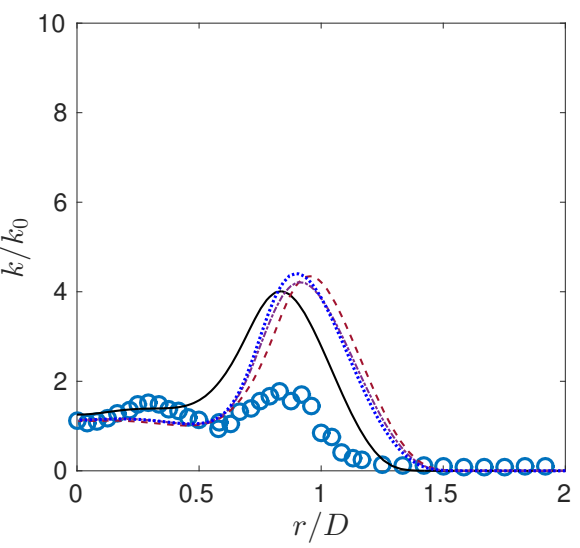

(b) $y / D \approx 4.5$

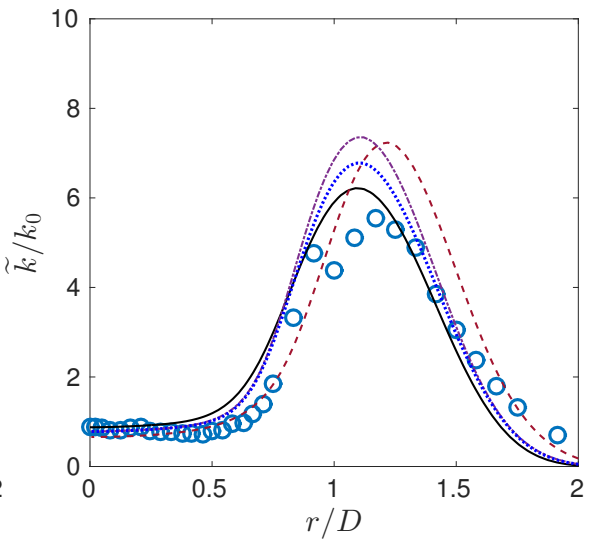

(d) $y / D \approx 8.5$

Fig. 16 Reacting flow turbulent kinetic energy profiles for the Bunsen flame calculation. 


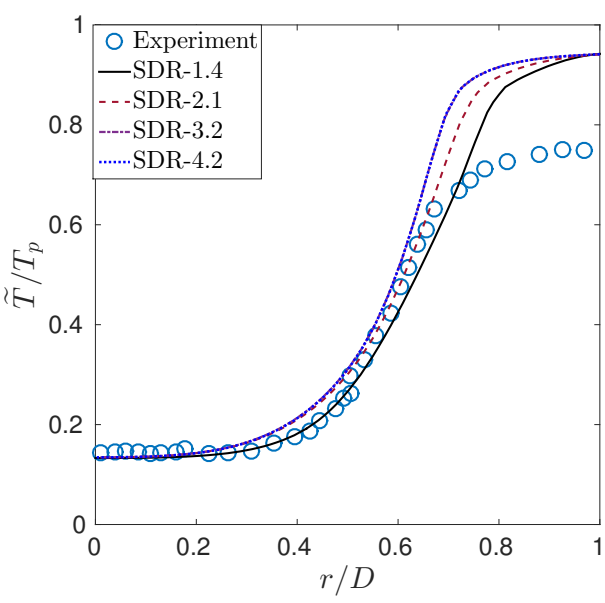

(a) $y / D \approx 2.5$

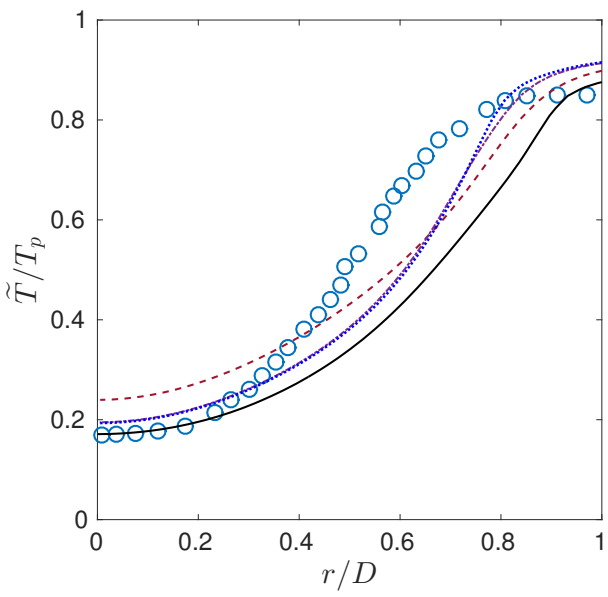

(c) $y / D \approx 6.5$

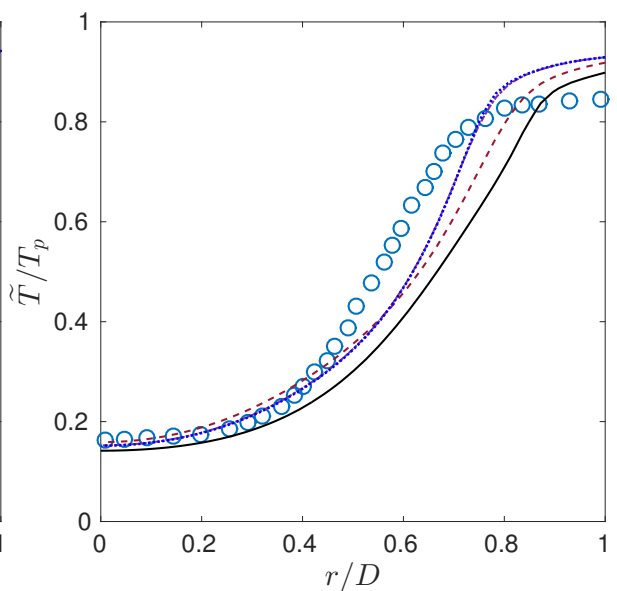

(b) $y / D \approx 4.5$

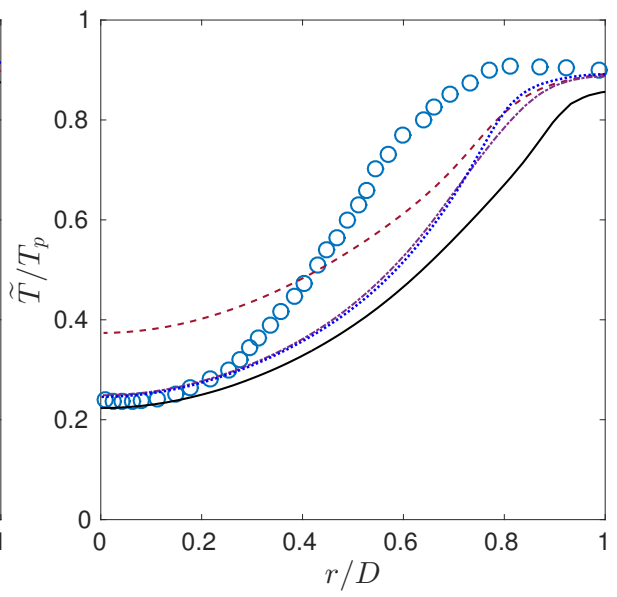

(d) $y / D \approx 8.5$

Fig. 17 Temperature predictions from different models for the Bunsen flame calculation. $T_{P}=2248 K$ is the adiabatic flame temperature. 


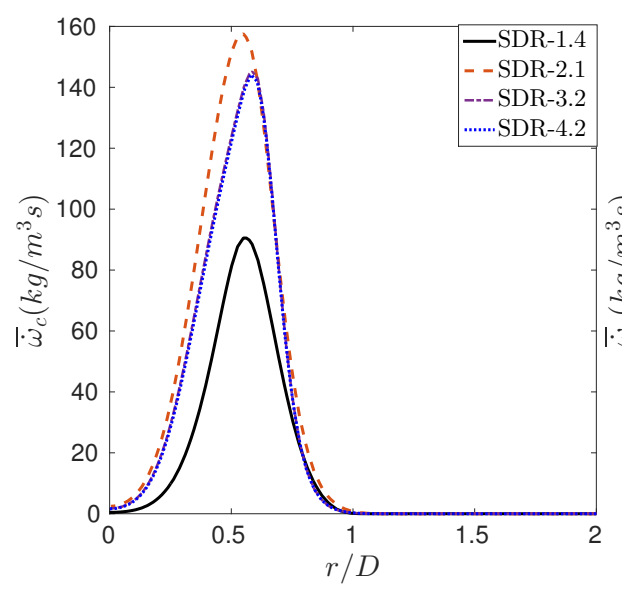

(a) $y / D \approx 2.5$

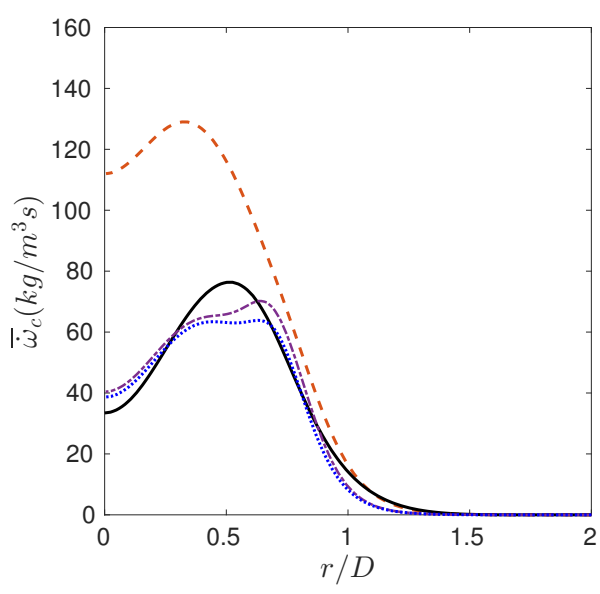

(c) $y / D \approx 6.5$

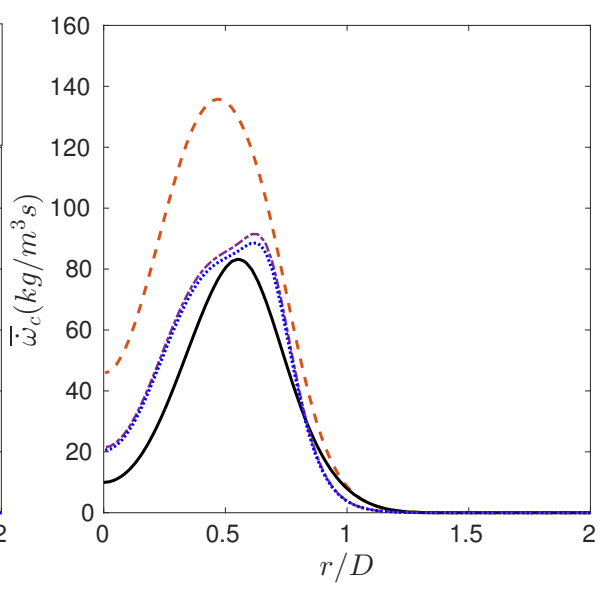

(b) $y / D \approx 4.5$

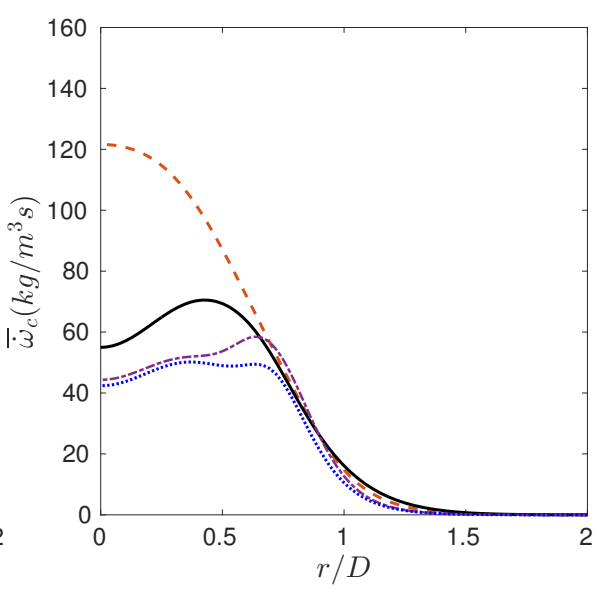

(d) $y / D \approx 8.5$

Fig. 18 Reaction rate prediction from different models for the Bunsen flame calculation. 


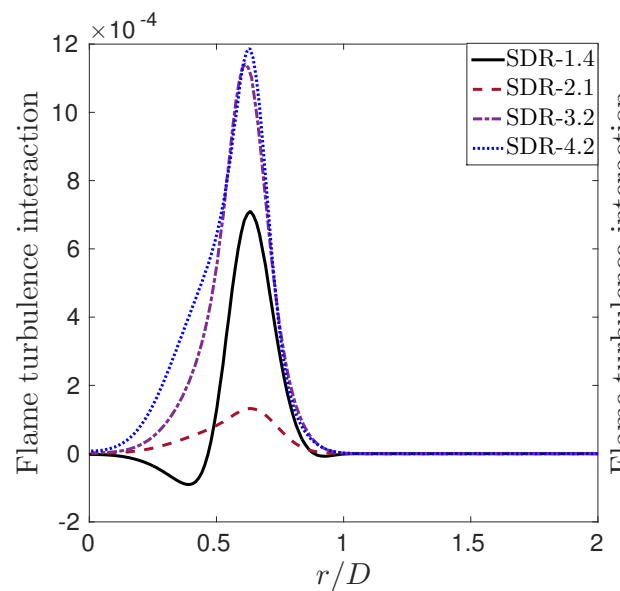

(a) $y / D \approx 2.5$

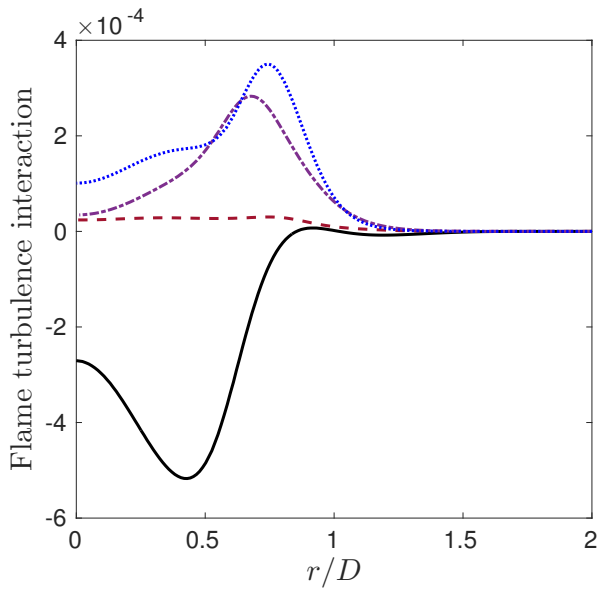

(c) $y / D \approx 6.5$

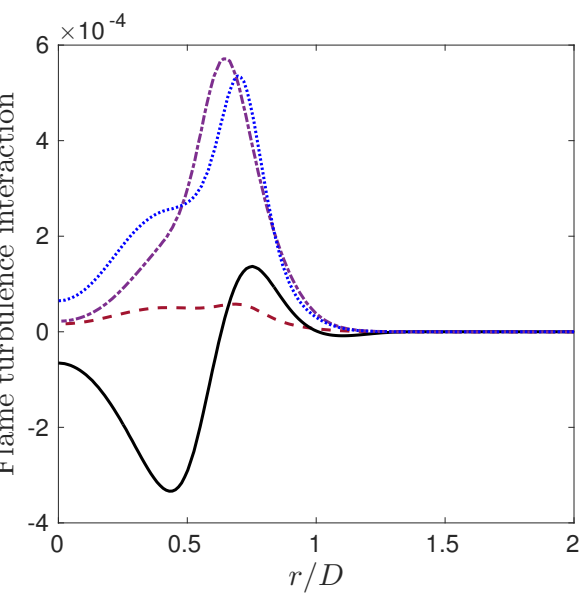

(b) $y / D \approx 4.5$

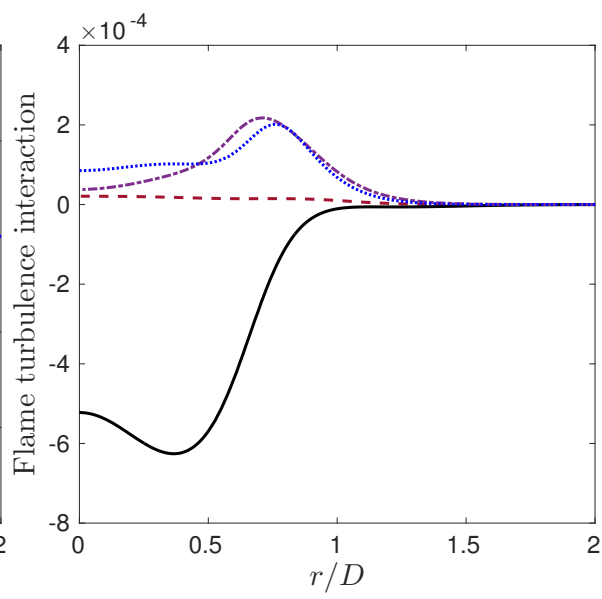

(d) $y / D \approx 8.5$

Fig. 19 Flame turbulence interaction $\left(\overline{\rho \alpha \nabla c^{\prime \prime} . \mathbf{S}^{\prime \prime} . \nabla \mathbf{c}^{\prime \prime}}\right)$ prediction from different models for the Bunsen flame calculation. The values are normalised using the respective $\rho_{u}, \delta_{L}^{0}$ and $u_{L}^{0}$. 

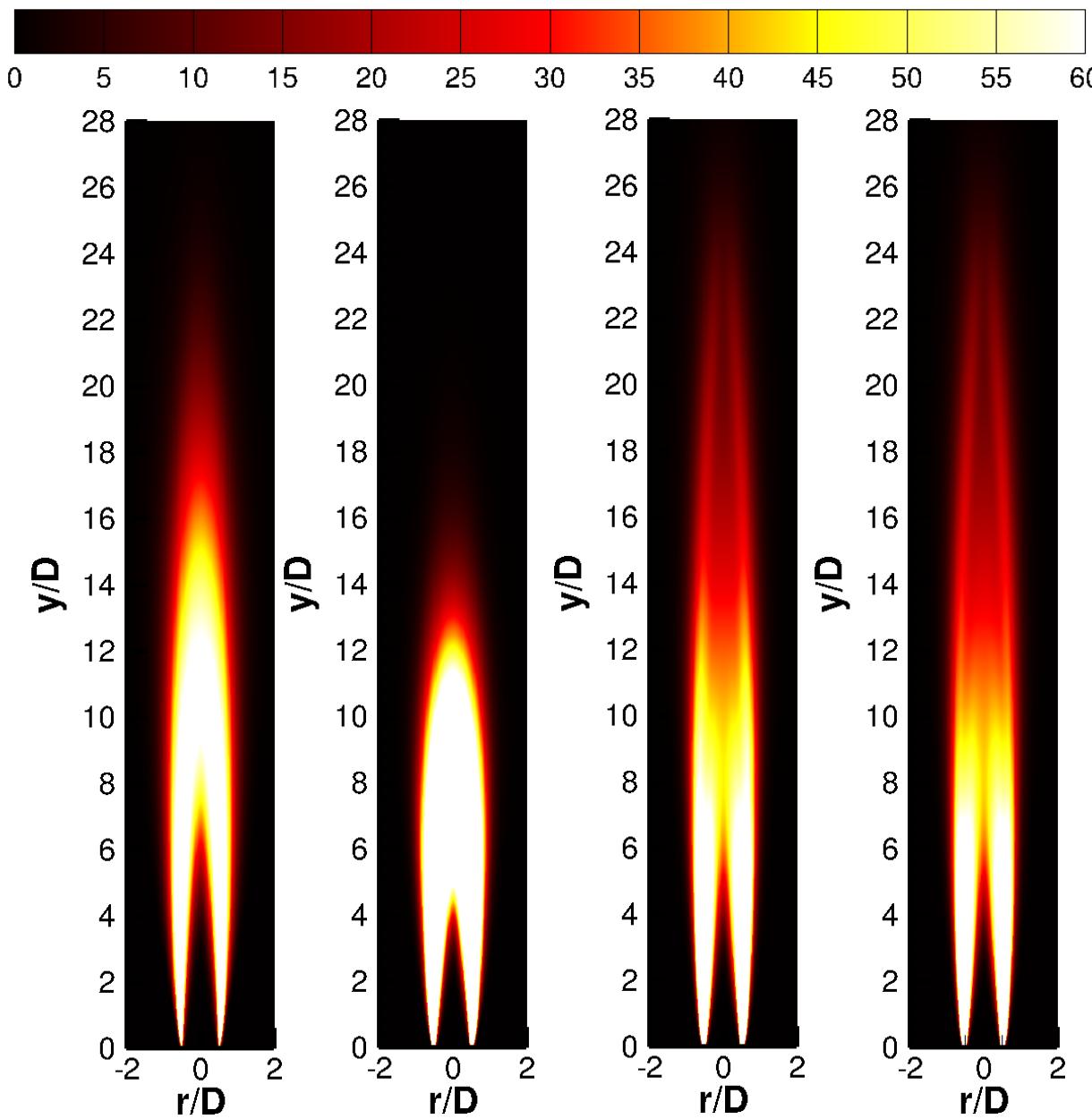
(a) SDR-1.4
(b) SDR-2.1
(c) SDR-3.2
(d) SDR-4.2

Fig. 20 Predicted reaction rate $\overline{\dot{\omega}}_{c}\left(\mathrm{~kg} / \mathrm{m}^{3} \mathrm{~s}\right)$ from different models for the Bunsen flame calculation. 


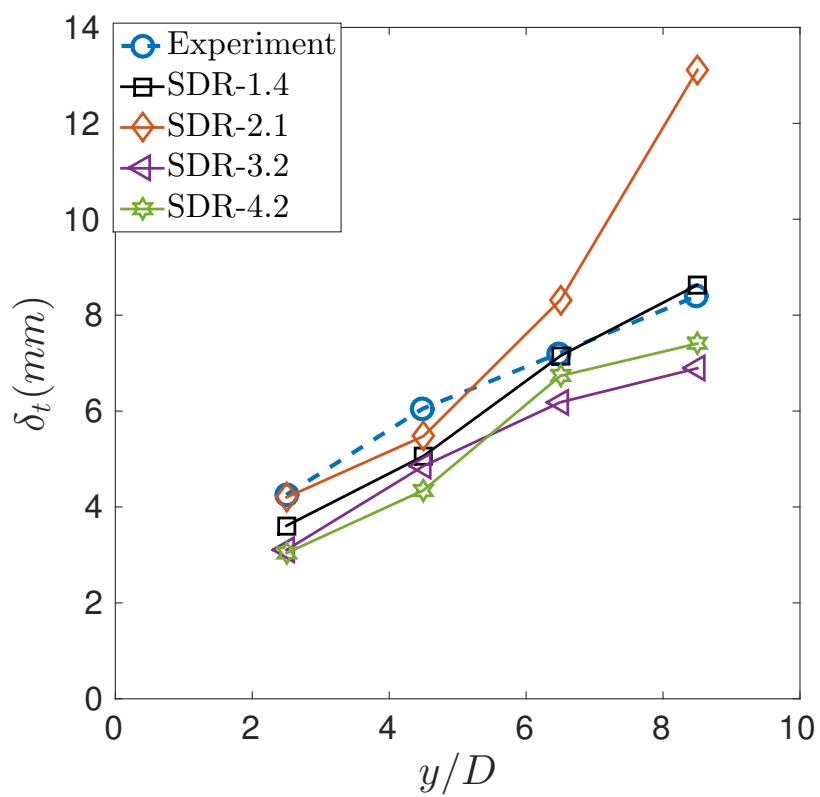

Fig. 21 Predicted axial variation of turbulent flame brush thickness in the Bunsen flame calculation

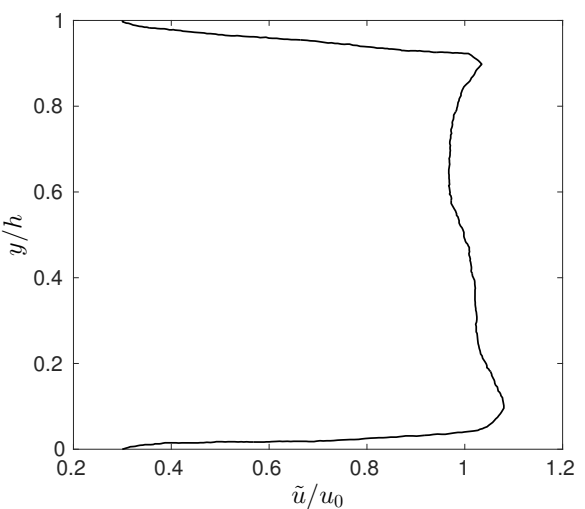

(a) Mean velocity profile

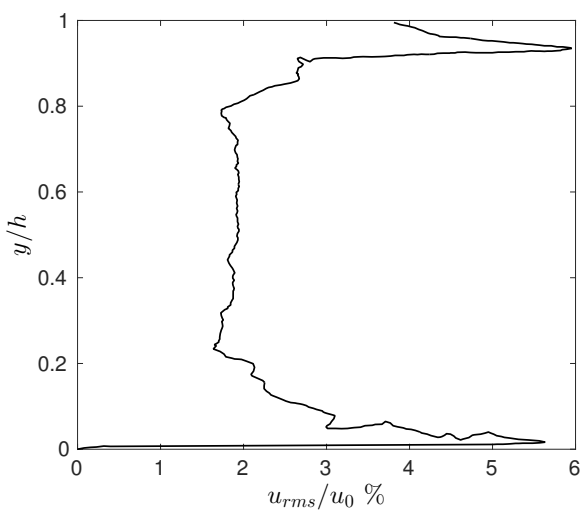

(b) Turbulence intensity profile

Fig. 22 Inlet profiles for the dump combustor simulation [55]. 


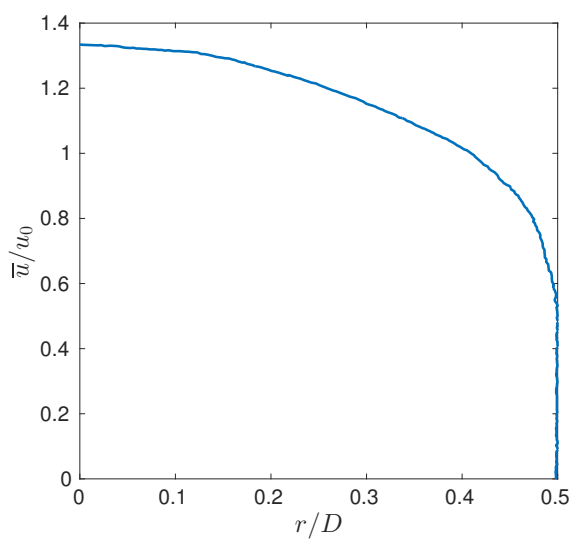

(a) Mean velocity profile

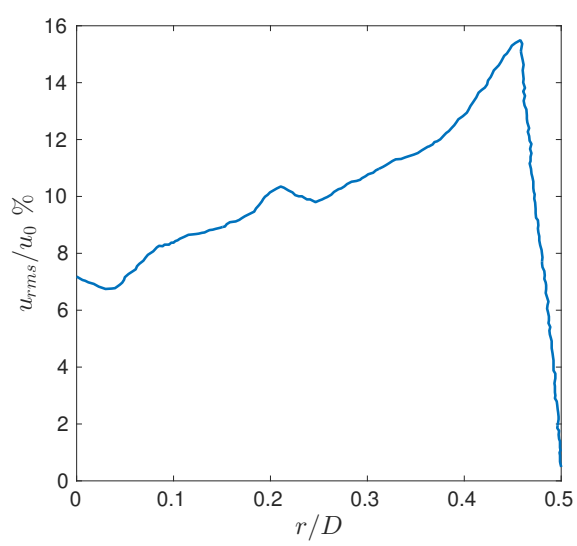

(b) Turbulence intensity profile

Fig. 23 Inlet profiles for the Bunsen flame simulation [22].

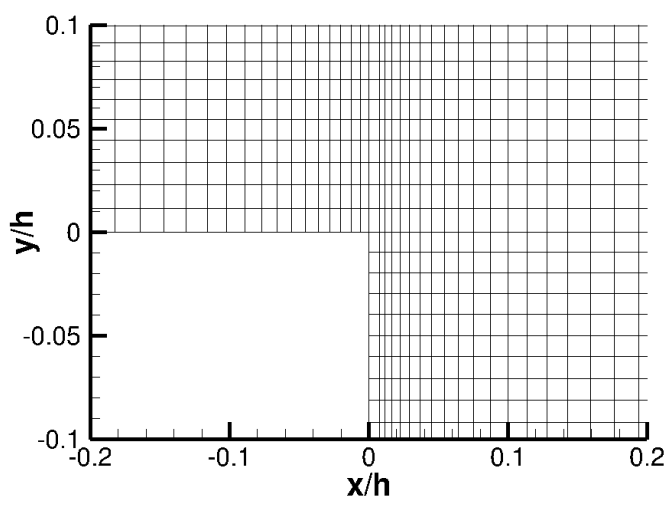

(a) Mesh near the backward facing step for $k-\epsilon$ model, $y^{+} \approx 30$ at the wall

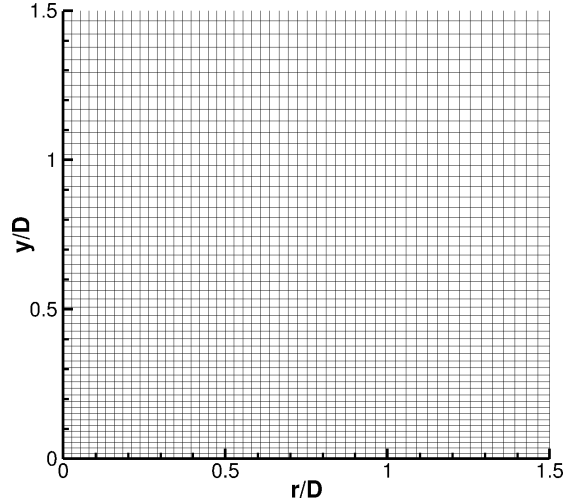

(b) Mesh near the inlet of the air-fuel mixture for the Bunsen flame simulation.

Fig. 24 Meshes used for the simulations. 


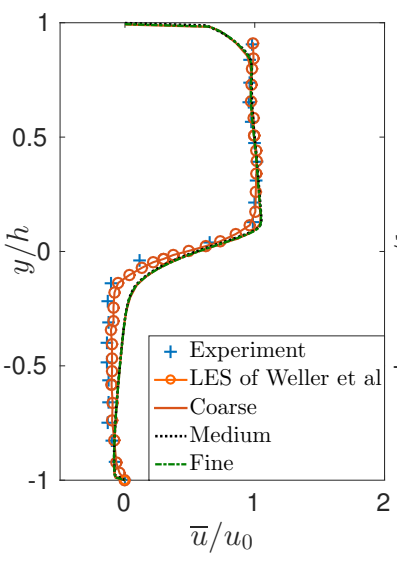

(a) $x / h=1$

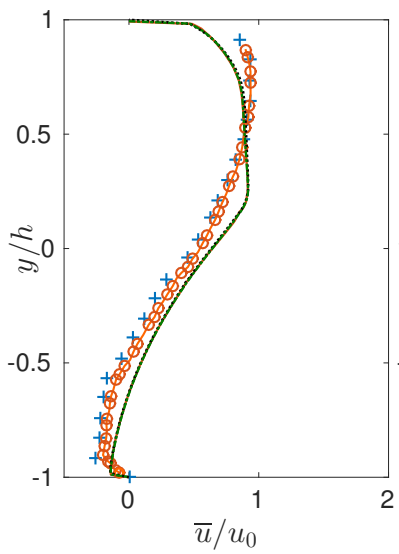

(c) $x / h=5$

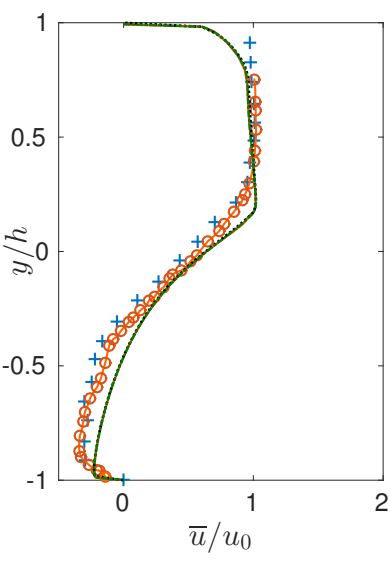

(b) $x / h=3$

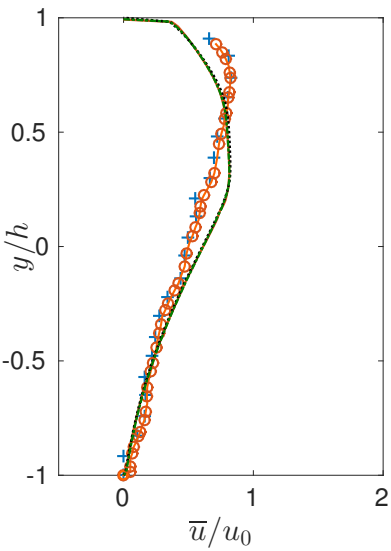

(d) $x / h=7$

Fig. 25 Isothermal flow velocity profiles for the dump combustor case by using three different meshes. 


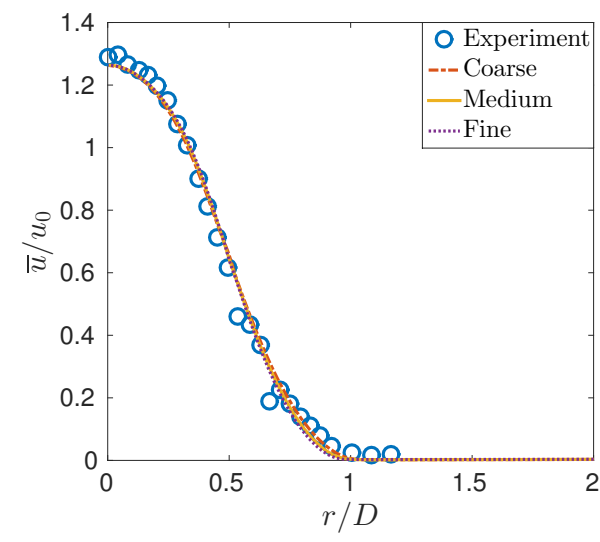

(a) $y / D \approx 2.5$

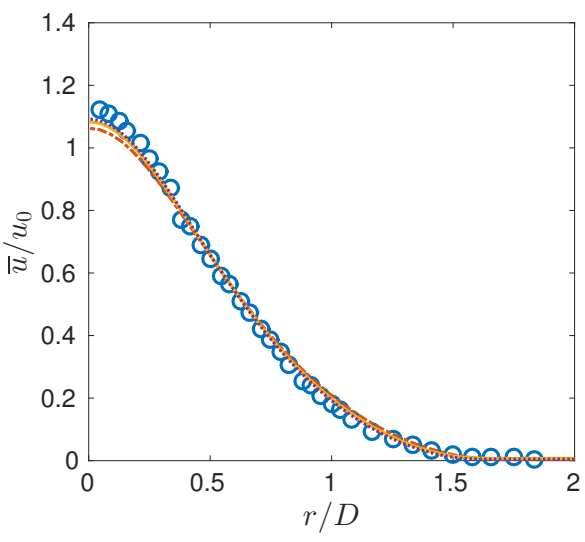

(c) $y / D \approx 6.5$

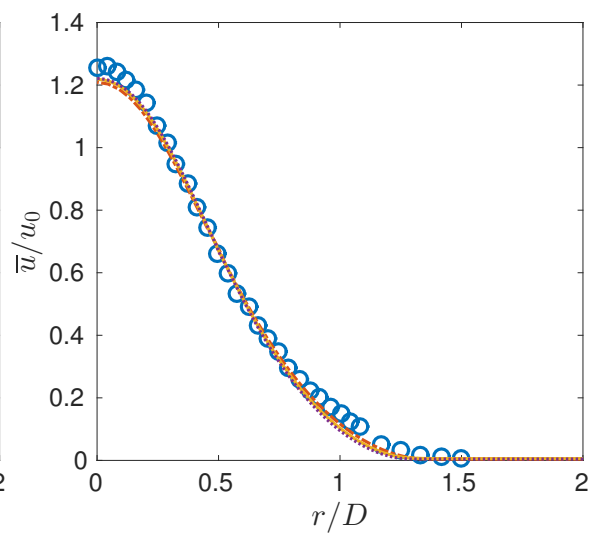

(b) $y / D \approx 4.5$

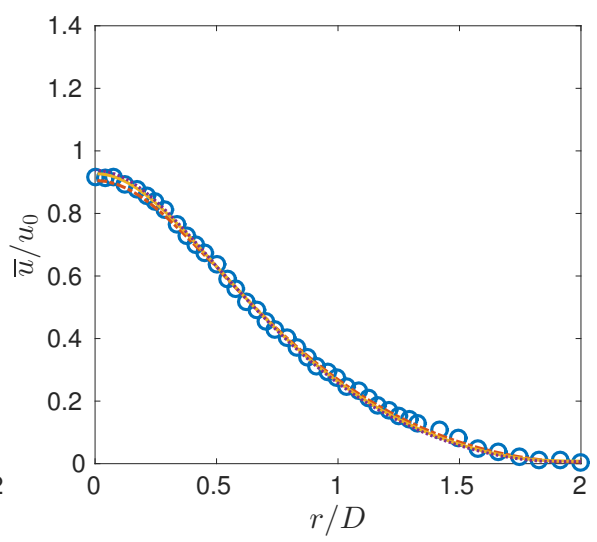

(d) $y / D \approx 8.5$

Fig. 26 Isothermal flow velocity profiles for the Bunsen flame case by using three different meshes. 